\title{
Boundary-reducing surgeries and bridge number
}

\author{
Kenneth L. Baker $\quad$ R. Sean Bowman John Luecke
}

July 31,2021

\begin{abstract}
Let $M$ be a 3-dimensional handlebody of genus $g$. This paper gives examples of hyperbolic knots in $M$ with arbitrarily large genus $g$ bridge number which admit Dehn surgeries which are boundary-reducible manifolds.
\end{abstract}

\section{Introduction}

A 3-dimensional handlebody of genus $g$ is a 3-ball with $g$ one-handles attached. In general, when the manifolds before and after Dehn surgery on a knot are homeomorphic, the surgery is called cosmetic. This paper studies cosmetic surgeries on a handlebody. Let $K$ be a knot in a genus $g$ handlebody $M$. When does $K$ admit a cosmetic surgery, i.e. a Dehn surgery which is again a genus $g$ handlebody?

When a knot $K$ in any 3 -manifold $M$ is isotopic into the boundary of $M$, then $K$ admits infinitely many surgeries that are manifolds homeomorphic to $M$ - infinitely many cosmetic surgeries. In particular, when $M$ is a handlebody of genus $g$, one obtains cosmetic surgeries on any knot parallel into the boundary of $M$. When $g=0$, GL89 shows that this is the only way a cosmetic surgery arises. When $g=1$, Berge and Gabai (Ber91, Gab89, Gab90]) give a classification of knots which admit cosmetic surgeries. Not all are isotopic into the boundary, but, if not, they show that they have bridge number one in the solid torus. Indeed, showing that the bridge number is one is an important part of the classification of these knots. Berge and Gabai also give ([Ber, Gab90]) examples of knots in handlebodies of genus $g \geq 2$ which admit cosmetic surgeries but which are not isotopic into the boundary of the handlebody. These turn out also to have bridge number one in the the handlebody. In Wu93, (see also Gab90, Question 4.5] and [Ber]), Wu conjectures that this is always the case - knots in handlebodies admitting cosmetic surgeries are always either isotopic into the boundary (i.e. bridge number zero) or bridge number one. However, Bowman gives counterexamples to this conjecture in [Bow13], exhibiting knots in a genus 2 handlebody that admit cosmetic surgeries but have bridge number greater than one. In Theorem 1.2(1), we generalize Bowman's result in two directions: (a) we show that certain infinite subcollections of the knots in Bow13 in fact have arbitrarily large bridge number (in particular, see Corollary 7.3 , and $(b)$ we give such infinite families for handlebodies of arbitrary genus. For a knot $K$ in a genus $g$ handlebody, $b_{g}(K)$ denotes the genus $g$ bridge number of $K$.

A compact 3-manifold is said to be boundary-reducible if it contains a properly embedded disk whose boundary is essential in the boundary of the manifold, and boundary-irreducible otherwise. There has been much interest in the question: when does a knot in a handlebody admit a Dehn surgery which is a boundaryreducible manifold? (see [CGLS87, Gab90, Question 4.5], and [Wu92b, Wu93]). Note that a handlebody of genus $g>0$ is boundary-reducible, so the cosmetic surgeries above are examples of such surgeries. For genus 1 handlebodies, the knots which admit such surgeries are classified ([Ber91, Gab89, Gab90], and [Sch90] for the reducible case), and in particular the only hyperbolic knots which admit a boundary-reducible surgery are 1-bridge in the solid torus. In Theorem 1.2(2) we give, for any genus $g>1$, infinite families of hyperbolic knots with arbitrarily large bridge number that admit Dehn surgeries giving a Seifert fiber space with $g-1$ one-handles attached (hence a boundary-reducible manifold). 
Definition 1.1. A $D(p, q)$-Seifert space is a Seifert fiber space over the disk with two exceptional fibers of order $p, q$.

Theorem 1.2. Let $M$ be a handlebody of genus $g>1$. For every positive integer $N$ the following hold.

1. There are infinitely many knots $K \subseteq M$ such that $K$ admits a nontrivial handlebody surgery and

$$
b_{g}(K) \geq N \text {. }
$$

Furthermore, the knots may be taken to have the same genus $g$ bridge number.

2. There are infinitely many pairs of relatively prime integers $p$ and $q$ such that for each pair, there are infinitely many knots $K \subseteq M$ admitting a surgery yielding a $D(p, q)$-Seifert space with $(g-1)$ one-handles attached. Furthermore, for each such $K$

$$
b_{g}(K) \geq N .
$$

Finally, fixing $(p, q)$, the knots may be taken to have the same genus g bridge number.

The knots in each family above have exteriors in $M$ which are irreducible, boundary-irreducible, atoroidal, and anannular. The slope of each surgery is longitudinal, that is, intersecting the meridian once, and there is only one nontrivial surgery on each knot which is a boundary-reducible manifold.

This is Theorem 6.8, proven in section 6

If one does not worry about establishing a lower bound on bridge number, the construction for Theorem 1.2 generalizes to give more examples of knots in a genus $g$ handlebody upon which Dehn surgery gives boundaryreducible manifolds.

Theorem 1.3. Let $M$ be a handlebody of genus $g>1$ and $p, q$ be non-zero, relatively prime integers. There are infinitely many knots $K \subseteq M$ admitting a longitudinal surgery yielding a $D(p, q)$-Seifert space with $(g-1)$ one-handles attached. Furthermore, the exterior in $M$ of each knot is irreducible, boundaryirreducible, atoroidal, and anannular. Finally, for fixed $p, q$, these knots can be taken to have the same genus $g$ bridge number in $M$.

This is Theorem 6.10, proven in section 6 .

Note that in Theorem 1.2(1), we show that the cosmetic surgery on these knots with large bridge number is unique. There are examples of knots in handlebodies that admit more than one cosmetic surgery and whose exteriors are irreducible, boundary-irreducible, atoroidal, and anannular, see (Berge [Ber, Frigerio et. al. [FMP03]). Note that by [Wu92b], any such knot will admit at most two cosmetic surgeries. Knots admitting more than one cosmetic surgery should be much more special, and in fact the only examples known to date have bridge number one. So we ask the following:

Question 1.4. Let $K$ be a knot in a handlebody whose exterior is irreducible, boundary-irreducible, atoroidal, and anannular. If $K$ admits more than one cosmetic surgery, must it have bridge number one in the handlebody?

The surgery dual knot to a Dehn surgery is the knot in the resulting manifold that is the core of the attached solid torus. Theorem 1.2(1) gives families of knots in a handlebody with large bridge number and that admit cosmetic surgeries. The surgery dual knots to these knots are also knots in handlebodies admitting cosmetic surgeries, and we can ask about their bridge number. Wu shows in Theorem 5 of Wu93 that a knot in a handlebody with a cosmetic surgery has bridge number at most one if and only if its surgery dual does too. Thus the surgery duals in the families given above will have bridge number greater than one.

Question 1.5. Is there a family of knots in a handlebody with the following properties?

1. Each knot admits a cosmetic surgery.

2. The bridge numbers for these knots and for their surgery duals grow without bound. 
3. The exterior of each knot in the family is irreducible, boundary-irreducible, atoroidal, and anannular.

The paper is organized as follows. In section 2 we define a collection of knots in a genus 2 handlebody, $H$. In section 3, we glue this genus 2 handlebody to a genus $g$ handlebody, $H^{\prime}$, along a 3 -punctured sphere that has nice properties in both $H$ and $H^{\prime}$. This produces a genus $g$ handlebody $M$, and the knots defined for $H$ become the knots in $M$. In section 4 , we show how twisting a knot along an annulus can increase the hitting number of the knot with surfaces of fixed Euler chararcteristic in $M$. In section 5, we use twisting along an annulus to produce infinitely many hyperbolic knots in $M$ which admit handlebody and boundary-reducible surgeries. In section 6, we use twisting along an annulus to find infinite families of knots among those from section 5 that also have large bridge number. In section 7, we clarify the connection between the knots constructed in this paper and the knots constructed in [Bow13.

\section{The little handlebody}

In this section we construct a family of knots in the genus 2 handlebody $P \times I$, where $P$ is a pair of pants (that is, a 2 -sphere minus three disjoint open disks) and $I=[-1,1]$. The surface $P \times\{1\}$ is $\partial$-incompressible in the complement of the knots, but becomes $\partial$-compressible after some nontrivial surgery. We call this handlebody the little handlebody in contrast with the big handlebody of Section 3 . The big handlebody is constructed by gluing the little handlebody to another handlebody along $P \times\{-1\}$.

We consider two knots in a 3-manifold $M$ to be the same if there is a homeomorphism of $M$ to itself taking one knot to the other.

Definition 2.1. Let $M$ be a 3-manifold with boundary. A properly embedded disk in $M$ is said to be essential if its boundary does not bound a disk in $\partial M$. When $M$ is a handlebody, such a disk is called a meridian disk of $M$. A properly embedded annulus is said to be essential if it is incompressible and not parallel into $\partial M$.

Definition 2.2. When $L$ is a 1 -manifold properly embedded in a 3 -manifold, $M$, we write $M_{L}$ to denote the exterior of $L$ in $M, \overline{M \backslash N(L)}$.

Fix a once-punctured torus $T$. Let $\mu$ and $\lambda$ be two oriented simple closed curves intersecting transversally in one point, so that the homology classes of $\mu, \lambda$ form a right-handed basis for $H_{1}(T)$. If $\alpha$ and $\beta$ are oriented simple closed curves in $T$, let $\alpha+\beta$ denote the simple closed curve homologous to $[\alpha]+[\beta]$. Define $\nu$ to be the simple closed curve $\lambda+\mu$.

Definition 2.3. Let $\tau$ be an unoriented, non-trivial simple closed curve in $T$. Then $\tau^{\prime}$ is defined to be a properly embedded arc in $T$ whose complement is an annulus with core $\tau$. We also write $\tau(p, q)$ when $\tau$ is homologous in $T$ to $\pm(p[\mu]+q[\lambda])$.

Note that by proper isotopies, the $\operatorname{arcs} \lambda^{\prime}, \mu^{\prime}$, and $\nu^{\prime}$ may be made mutually disjoint in $T$. Also we may write $\mu=\tau(1,0), \lambda=\tau(0,1)$, and $\nu=\tau(1,1)$. We will also consider the curves $\lambda-\mu=\tau(-1,1), \lambda+\nu=\tau(1,2)$, and $\mu+\nu=\tau(2,1)$.

Definition 2.4. Consider the genus two handlebody $H \cong T \times I$, with $I=[-1,1]$. Let $\tau$ be an essential simple closed curve in $T$. Define the meridian disk of $H, D_{\tau}=\tau^{\prime} \times I$. Define annuli $A_{-}(\tau)=\tau \times[-1,-1 / 2]$, $\widehat{R}(\tau)=\tau \times[-1 / 2,1 / 2]$, and $A_{+}(\tau)=\tau \times[1 / 2,1]$. Define $K(\tau)$ to be the knot $\tau \times\{0\}$ in $H$. We refer to the components of $\partial \widehat{R}(\tau)$ as $L_{ \pm}(\tau)$ so that $L_{+}(\tau)=\tau \times\{1 / 2\}$ and $L_{-}(\tau)=\tau \times\{-1 / 2\}$. Note that $D_{\tau}$ is disjoint from $L_{ \pm}(\tau)$.

We will be looking at the knots above as obtained by twisting along an annulus. 
Definition 2.5. Let $K$ be a knot and $R$ be an annulus in the interior of $H$. Assume $K$ intersects $R$ transversely. Let $R \times[0,1]$ be a product neighborhood of $R$ in $H$. Let $h_{n}: R \times[0,1] \rightarrow R \times[0,1]$ be a homeomorphism gotten by $i$ complete twists along $R$, where $h_{n}$ is the identity on $R \times\{0,1\}$. Define the knot $K$ twisted $n$ times along $R$ as $[K-(R \times[0,1])] \cup h_{n}(K \cap(R \times[0,1]))$. See [BGL13] for a more detailed description.

Definition 2.6. Let $\tau, \kappa, \alpha$ be essential simple closed curves in $T$ with $\kappa$ and $\alpha$ not isotopic in $T$. If $\tau$ is the result of $n$ positive Dehn twists of $\kappa$ along $\alpha$ we indicate this by writing $\tau(\kappa, \alpha, n)$ for $\tau$. Let $\mathcal{L}(\kappa, \alpha)$ be the link $K(\kappa) \cup L_{+}(\alpha) \cup L_{-}(\alpha)$ in $H$. In the notation above, $K(\tau(\kappa, \alpha, n))$ is the knot $K(\kappa)$ twisted $n$ times along the annulus $\widehat{R}(\alpha)$, where the sign of the twisting is chosen to agree with a positive Dehn twist.

In twisting $K(\kappa)$ along $\widehat{R}(\alpha)$, we will always assume that $\kappa$ and $\alpha$ are not isotopic in $T$ and are taken to intersect minimally in $T$. Let $\mathcal{L}=\mathcal{L}(\kappa, \alpha)$, and let $T_{+}, T_{-}$, and $T_{K}$ be the boundary components of $H_{\mathcal{L}}$ corresponding to $L_{+}(\alpha), L_{-}(\alpha)$, and $K(\kappa)$, respectively. By abuse of notation, we will also refer to the part of $A_{+}(\alpha)$ and $A_{-}(\alpha)$ lying in $H_{\mathcal{L}}$ as $A_{+}(\alpha)$ and $A_{-}(\alpha)$, respectively. These are also annuli, and they are essential since $L_{+}$and $L_{-}$are nontrivial in $H$. Let $R$ be the planar surface $\widehat{R}(\alpha) \cap H_{\mathcal{L}}$. This surface has one longitudinal boundary component on each of $T_{+}$and $T_{-}$and one or more coherently oriented meridional boundary components on $T_{K}$. It is also incompressible in $H_{\mathcal{L}}$ since compression would show that either $L_{+}$ or $L_{-}$is trivial or there is a non-separating sphere in $H$.

The knots obtained by twisting $K(\kappa)$ along $\widehat{R}(\alpha)$ are of the type studied in Bow13. Lemmas 2.8, 2.10. 2.11 , 2.13, 3.9. 3.10, and 3.6 essentially appear there (in whole or in part) and are reproduced here for completeness.

Lemma 2.7. Let $\tau$ be an essential simple closed curve in $T$. Then $K(\tau)$ is a core curve of the genus 2 handlebody $H \cong T \times I$.

Proof. Since $T \backslash \tau^{\prime}$ is an annulus with core curve $\tau$, we have that $H \backslash D_{\tau} \cong\left(T \backslash \tau^{\prime}\right) \times I$ is a solid torus with $K(\tau)$ as its core. Because $H$ is recovered by attaching a 1-handle to this solid torus (reversing the chopping along $D_{\tau}$ ) we have our result.

Lemma 2.8. Let $W$ be the compression body that is the exterior of a core curve of a genus 2 handlebody. $U p$ to isotopy, there is a unique non-separating compressing disk for $W$. In particular, up to isotopy, $D_{\tau}$ is the unique non-separating meridian disk for $H$ which does not meet $K(\tau)$.

Proof. Let $\widehat{T}$ be the (closed) torus. We may view $W$ as a thickened torus $\widehat{T} \times I$ with a 1 -handle attached to one side. Let $D$ be the cocore of this 1-handle. Among all non-separating compressing disks for $W$ that are not isotopic to $D$, let $E$ be one that intersects $D$ minimally.

If $E$ is disjoint from $D$, then we may isotop $E$ into $\widehat{T} \times I$ disjoint from the feet of the 1 -handle. Since $\widehat{T} \times I$ is irreducible and $\partial$-irreducible, the disk $E$ is $\partial$-parallel and hence separating in $\widehat{T} \times I$. In order for $E$ to be non-separating in $W$, the disk $\partial E$ bounds in $\partial(\widehat{T} \times I)$ must contain exactly one of the feet of the 1 -handle. But then $E$ is isotopic to $D$ in $W$.

Thus $E$ is not disjoint from $D$. Let $c$ be an outermost arc of intersection in $D$ and let $D^{\prime}$ be the subdisk it cuts off. Surger $E$ along $D^{\prime}$ to produce disjoint disks $E_{1}$ and $E_{2}$ that intersect $D$ fewer times than $E$. Each must be either separating or isotopic to $D$. As $E_{1} \cup E_{2}$ must also be non-separating, we may assume $E_{1}$ is isotopic to $D$ and $E_{2}$ is separating. If $E_{2}$ were $\partial$-parallel then $E$ is isotopic to $D$, a contradiction. Hence $\partial E_{2}$ must separate $\partial W$ into two punctured tori, one of which contains $\partial E_{1}$. But then banding $\partial E_{2}$ to $\partial E_{1}$ to give $\partial E$ shows that $\partial E$ is isotopic to $\partial E_{1}$ which in turn is isotopic to $\partial D$. Since $W$ is irreducible, $E$ must be isotopic to $D$, a contradiction.

Lemma 2.7 then gives the final statement. 
Choose a subarc $\nu_{0}$ of $\partial D_{\nu}$ such that $\nu_{0} \cap(\mu \times\{-1\} \cup \lambda \times\{1\})=\partial \nu_{0}$ (there are two such choices).

Define the pair of pants $P$ to be a regular neighborhood of $\mu \times\{-1\}, \lambda \times\{1\}$, and $\nu_{0}$. See Figure 1 . Then $H \cong P \times I$ : cutting along the disjoint disks $D_{\mu}, D_{\lambda}$ gives the product of a disk and an interval - which reglues to give $P \times I$. In particular $P$ is incompressible in $H$. Let $\partial_{-} P$ be the boundary component of $P$ homotopic to $\mu \times\{-1\}$ in $\partial H, \partial_{+} P$ the component homotopic to $\lambda \times\{1\}$, and $\partial_{0} P$ the third component. See Figures 11(right) and 3 (left).
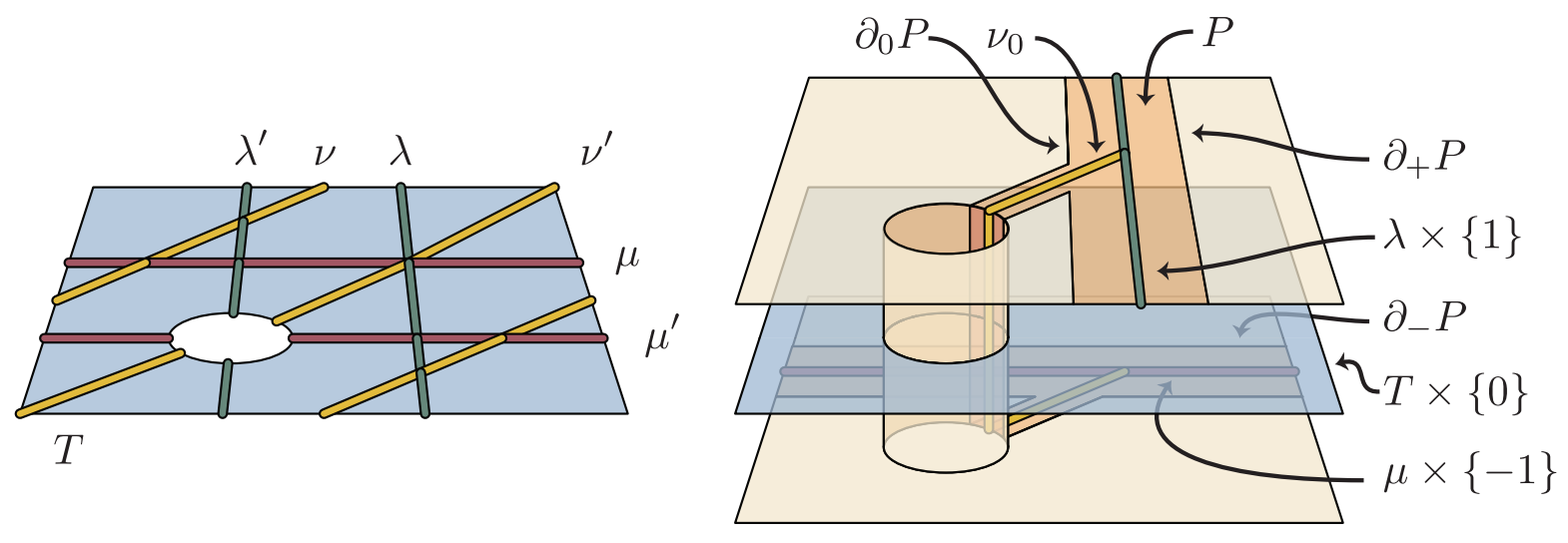

$H \cong T \times I$

Figure 1: (Left) The punctured torus $T$ with $\operatorname{arcs} \mu^{\prime}, \lambda^{\prime}, \nu^{\prime}$ and closed curves $\mu, \lambda, \nu$. (Right) The handlebody $H \cong T \times I$ with the curves $\mu^{\prime} \times\{-1\}$ and $\lambda^{\prime} \times\{1\}$ and arc $\nu_{0}$ in $\partial H$. Their regular neighborhood $P$ is also shown.

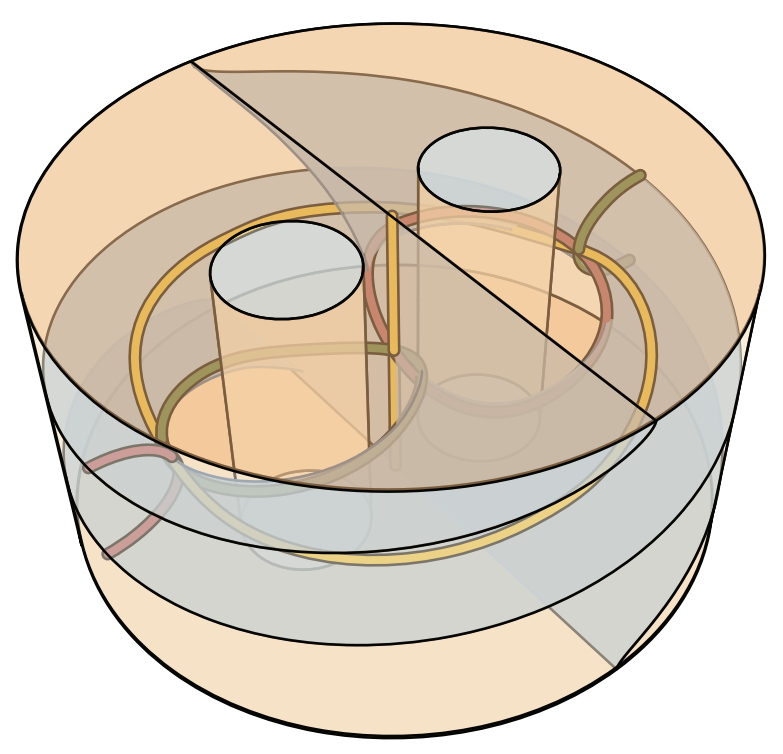

$H \cong P \times I \cong T \times I$

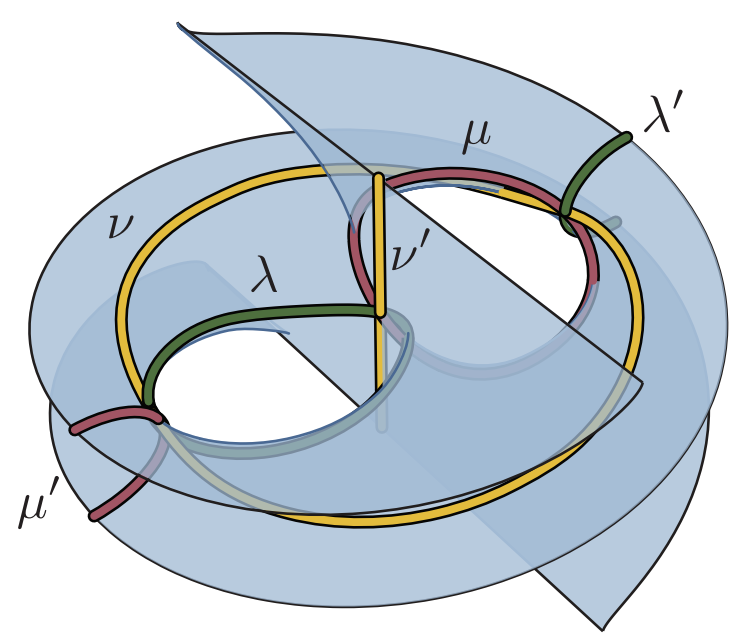

$T \times\{0\}$

Figure 2: (Left) A view of $H$ as $P \times I$. (Right) The surface $T \times\{0\} \subset H$ is isolated with its basic curves and arcs.

The disks $D_{\mu}, D_{\lambda}$, and $D_{\nu}$ are disjoint, non-separating $\partial$-compressing disks for $P$ in $H$. We give them alternate names according to which component of $\partial P$ they are disjoint from. In this way, we let $D_{-}=D_{\mu}$ 

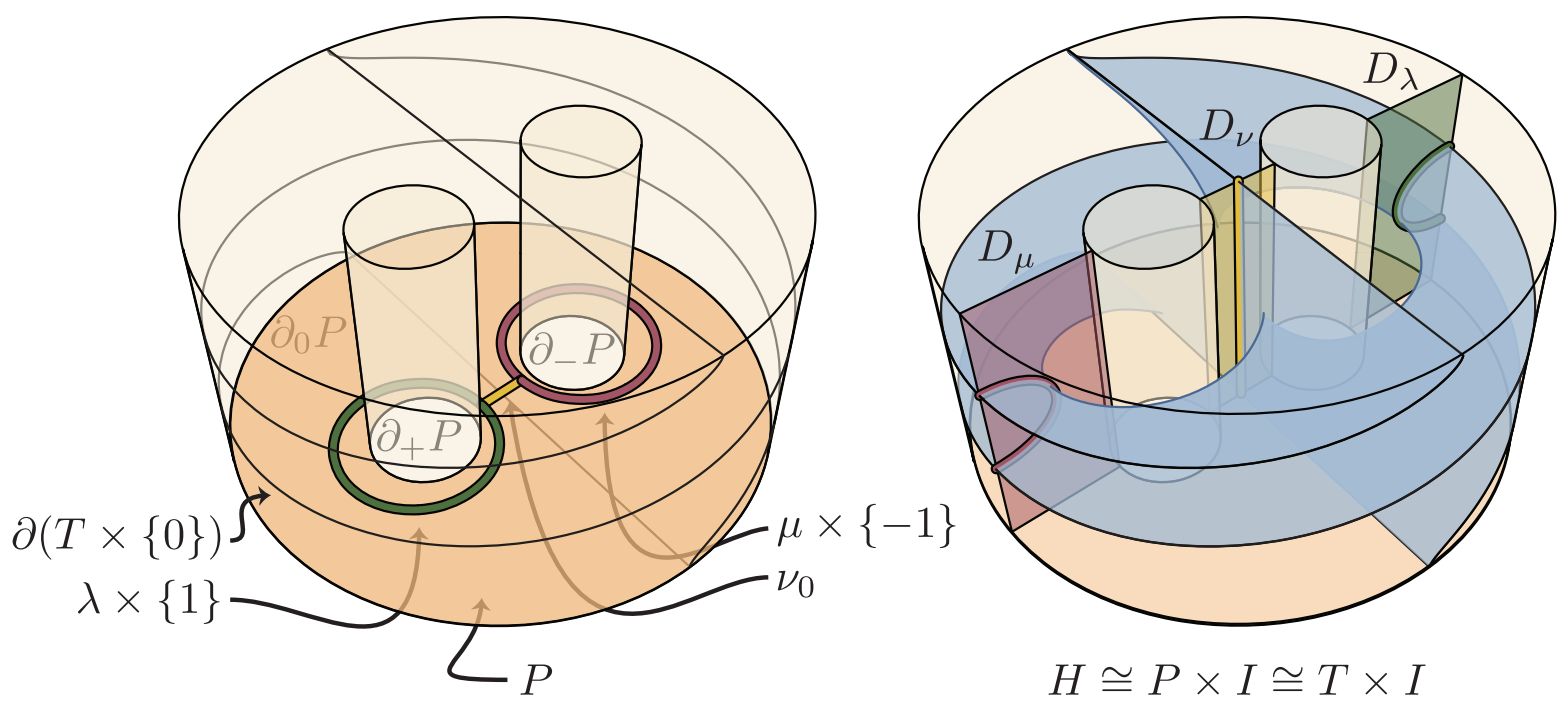

Figure 3: (Left) Omitting the top and front of this view of $\partial H$ reveals the surface $P$ and its defining curves from Figure 1. (Right) The disks $D_{\lambda}, D_{\mu}$, and $D_{\nu}$ are also shown relative to $T \times\{0\}$ in $H$.

so that $D_{-} \cap \partial_{-} P=\emptyset, D_{+}=D_{\lambda}$ so that $D_{+} \cap \partial_{+} P=\emptyset$, and $D_{0}=D_{\nu}$ so that $D_{0} \cap \partial_{0} P=\emptyset$. Since $H$ is homeomorphic to the product $P \times I$, the disks $D_{-}, D_{0}$, and $D_{+}$are also product disks with respect to this product structure and, up to isotopy, are the unique non-separating $\partial$-compressing disks for $P$ in $H$.

Definition 2.9. For properly embedded, connected submanifolds $x, y$, we use the notation $\Delta(x, y)$ for the geometric intersection number, the minimal number of intersections between $x$ and $y$ up to proper isotopy. If $x$ or $y$ has multiple components, we take $\Delta(x, y)$ to be maximum of this over their components. In the context of curves in a surface, especially tori, $\Delta(x, y)$ is also referred to as the distance of $x$ and $y$. For oriented submanifolds $x, y$ of an oriented manifold we use $x \cdot y$ for their algebraic intersection number. By isotoping $\tau$ in $T$ to intersect $\mu^{\prime}, \lambda^{\prime}, \nu^{\prime}$ coherently we get the following.

Lemma 2.10. Let $\tau$ be a simple closed curve in $T$.

- $\left|K(\tau) \cdot D_{\lambda}\right|=\left|K(\tau) \cdot D_{+}\right|=\Delta(\tau, \lambda)$,

- $\left|K(\tau) \cdot D_{\mu}\right|=\left|K(\tau) \cdot D_{-}\right|=\Delta(\tau, \mu)$, and

- $\left|K(\tau) \cdot D_{\nu}\right|=\Delta(\tau, \nu)$.

Proof. The intersection numbers occur in $H \cong T \times I$.

Lemma 2.11. If $D$ is a compressing disk for $H_{K(\tau)}$ such that $D \cap P$ is a single arc, then $\tau$ is isotopic to $\mu$, $\lambda$, or $\nu$ in $T$.

Equivalently, if $P$ is $\partial$-compressible in $H_{K(\tau)}$, then $\tau$ is isotopic to $\mu$, $\lambda$, or $\nu$ in $T$.

Proof. Since both $P$ and $\partial H \backslash P$ are incompressible in $H \cong P \times I$, the arc of $D \cap P$ must be essential in $P$. Then, fixing this arc, we may isotop $D \cup K(\tau)$ so that $D$ is a product disk in $H \cong P \times I$. Therefore $K(\tau)$ must be disjoint from one of the non-separating product disks $D_{\mu}, D_{\lambda}$, or $D_{\nu}$. By Lemma 2.10, $\tau$ may be isotoped in $T$ to be disjoint from $\mu, \lambda$, or $\nu$ correspondingly. Thus $\tau$ is isotopic to that curve.

Lemma 2.12. Assume $\tau$ is not isotopic to $\mu, \lambda$, or $\nu$ in $T$. If $D$ is a compressing disk for $H_{K(\tau)}$ such that $D \cap P$ is a pair of arcs $a_{1} \cup a_{2}$, then there are meridian disks $D_{1}, D_{2}$ such that $D_{i} \cap P=a_{i}$ with a banding disjoint from $P$ that produces $D$. 
Proof. Let $D$ be a compressing disk for $H_{K(\tau)}$ such that $D \cap P$ is a pair of $\operatorname{arcs} a_{1} \cup a_{2}$. By Lemma 2.11 these arcs must be essential in $P$.

Let $b_{1}$ and $b_{2}$ be two $\operatorname{arcs}$ in $P$ parallel to $a_{1}$ so that each component of $P \backslash\left(b_{1} \cup b_{2}\right)$ contains exactly one of $a_{1}$ or $a_{2}$. Let $F_{1}$ and $F_{2}$ denote the product disks $b_{1} \times I$ and $b_{2} \times I$, respectively, and choose $D$ to minimize $\left|D \cap\left(F_{1} \cup F_{2}\right)\right|$ subject to the constraint that $D \cap P$ consists of the two arcs $a_{1}$ and $a_{2}$. Isotop $K(\tau)$ to minimize $\left|K(\tau) \cap\left(F_{1} \cup F_{2}\right)\right|$, keeping $K(\tau)$ disjoint from $D$. Since $F_{1} \cup F_{2}$ separates a ball $B$ from $H$ containing $a_{1}$ but not $a_{2}, D \cap\left(F_{1} \cup F_{2}\right) \neq \emptyset$.

Let us assume, without loss of generality, that $D \cap F_{1} \neq \emptyset$. We may assume that there are no simple closed curves of intersection in $D \cap F_{1}$ since $H_{K(\tau)}$ is irreducible. Let $c$ be an arc of $D \cap F_{1}$ outermost in $F_{1}$ away from $b_{1}$ which cuts off a subdisk $F_{1}^{\prime}$ of $F_{1}$ whose interior does not meet $D$ or $b_{1}$.

Assume $c$ cuts off a subdisk $D^{\prime}$ of $D$ which is disjoint from $P$. Then $F_{1}^{\prime} \cup D^{\prime}$ is a disk disjoint from $P$, and thus it must be $\partial$-parallel in $H \cong P \times I$. Hence there is an isotopy of $D \cup K(\tau)$ fixing $D \cap P$ that reduces $\left|D \cap\left(F_{1} \cup F_{2}\right)\right|$. (We may surger $D$ along $F_{1}^{\prime}$ and further push off of $F_{1}^{\prime}$ to create a new disk that intersects $F_{1} \cup F_{2}$ fewer times than $D$. Since $F_{1}^{\prime} \cup D^{\prime}$ is boundary parallel, this surgery may be realized by an isotopy of $D^{\prime}$ to $F_{1}^{\prime}$.)

Therefore $c$ divides $D$ into two halves each containing one of $a_{1}$ or $a_{2}$. Surgering along $F_{1}^{\prime}$ we obtain two parallel $\partial$-compressing disks for $P$ in $H$ (they are both isotopic, relative to $P$, to product disks). Thus we have shown that $D$ consists of $D_{1}$ and $D_{2}$, isotopic to product disks, tubed along an arc in $\partial H \backslash P$.

Lemma 2.13. If $\tau \notin\{\lambda, \mu, \nu, \lambda-\mu, \lambda+\nu, \mu+\nu\}$, then the boundary of any compressing disk of $\partial H$ in $H_{K(\tau)}$ meets $P$ in at least 3 arcs.

Proof. Let $D$ be a compressing disk in $H_{K(\tau)}$, isotoped to minimize $|D \cap P|$. If $|D \cap P|=1$ then Lemma 2.11 implies that $\tau$ is $\mu, \lambda$, or $\nu$ (in which case $\{\Delta(\tau, \lambda), \Delta(\tau, \mu), \Delta(\tau, \nu)\}=\{0,1,1\}$ ). We now proceed to show that if $|D \cap P|=2$, then $\{\Delta(\tau, \lambda), \Delta(\tau, \mu), \Delta(\tau, \nu)\}=\{1,1,2\}$ as multisets. It is a simple exercise to see that this implies that $\tau$ is one of the curves $\lambda-\mu, \lambda+\nu$, or $\mu+\nu$.

Assuming that $|D \cap P|=2$, set $D \cap P=a_{1} \cup a_{2}$. Lemma 2.12 implies that $D$ is isotopic to the banding of product disks $a_{1} \times I, a_{2} \times I$ of $H \cong P \times I$ along an arc $\alpha$ disjoint from $P=P \times\{-1\}$. We may isotop $\alpha$ to be an arc in the interior of $P \times\{1\}$ meeting each $a_{1} \times\{1\}$ and $a_{2} \times\{1\}$ in its endpoints. Say such an arc $\alpha$ is $\partial$-parallel if $\alpha$ together with a subarc of each $a_{1} \times\{1\}, a_{2} \times\{1\}$, and $\partial P \times\{1\}$ bounds a rectangular disk $R$ in $P \times\{1\}$; this rectangle guides an isotopy of the disk $D$ obtained by banding of $D_{1}$ and $D_{2}$ along $\alpha$ that reduces $|D \cap P|$. Hence in our situation, the arc $\alpha$ cannot be $\partial-$ parallel.

Figure 4(Left) exhibits the four homeomorphism types of pairs of essential arcs $a_{1}, a_{2}$ embedded in $P$ along with the homeomorphism types of arcs $\alpha$ that connect $a_{1}$ and $a_{2}$. By inspection one sees that there is a single homeomorphism type of $\left(P, a_{1} \cup a_{2}, \alpha\right)$ for which $\alpha$ is not $\partial$-parallel; this is highlighted in Figure 4(Left). In particular $a_{1}$ and $a_{2}$ are non-isotopic arcs in $P$ that are each non-separating. Hence we must have that $D_{1}$ and $D_{2}$ are two of the three non-separating product disks $D_{\lambda}, D_{\mu}, D_{\nu}$ and $D$ is a non-separating disk obtained by banding them together in the manner indicated. This gives six possibilities for $D$, all equivalent to Figure 4(Right) by a level preserving homeomorphism of $P \times I$. Regardless of which of these six actually is $D$, since $K(\tau)$ is isotopic to the core curve of the solid torus $H \backslash D$ by Lemma 2.8 , we can then see that $K(\tau)$ intersects two of the disks $D_{\lambda}, D_{\mu}, D_{\nu}$ just once and the third disk twice as claimed.

\section{The big handlebody}

In this section we construct knots in handlebodies which have nontrivial surgeries yielding either handlebodies or Seifert fiber spaces with attached 1-handles. To get the originating handlebody, we glue the handlebody 

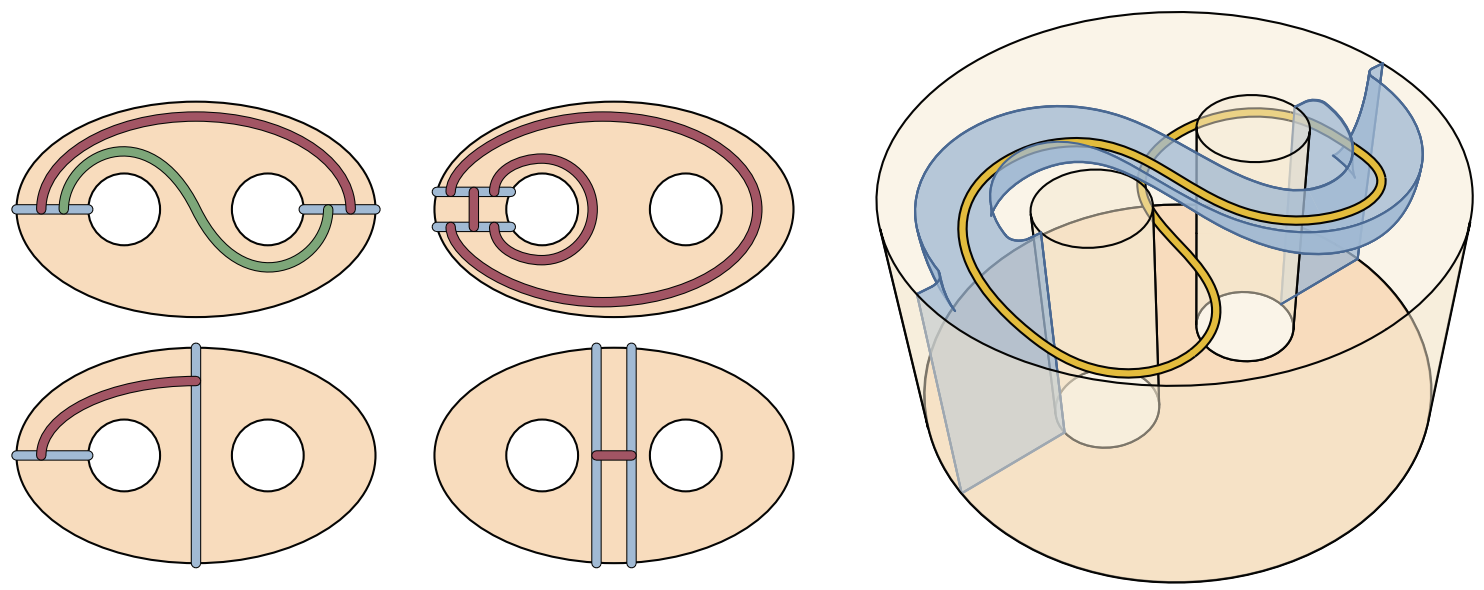

Figure 4: (Left) The four possibilities for $\left(P, a_{1} \cup a_{2}\right)$ up to homeomorphism. Each shows the possible arcs $\alpha$, up to homeomorphism, that join the $\operatorname{arcs} a_{1}$ and $a_{2}$. The one which is not $\partial$-parallel is in the top left. (Right) The compressing disk in $H_{K(\tau)}$ up to homeomorphism. The knot $K(\tau)$ intersects two of $D_{\lambda}, D_{\mu}$, and $D_{\nu}$ once and the other one twice. $\left(D_{\lambda}, D_{\mu}\right.$, and $D_{\nu}$ are the three non-separating product disks of $H \cong P \times I$.)

$H$ of the previous section to another handlebody along the pair of pants $P$. The knots of interest are those in $H$, of the preceding section, under this embedding of $H$.

Definition 3.1. A simple closed curve in the boundary of a handlebody is primitive if there is a meridian disk for the handlebody meeting the curve exactly once - we refer to such a disk as a primitivizing disk for the curve. Equivalently, a simple closed curve in the boundary of a handlebody is primitive if attaching a 2 -handle along it results in a handlebody.

Definition 3.2. Let $H^{\prime}$ be a genus $g>1$ handlebody. We say that a set $\left\{a_{1}, \ldots, a_{n}\right\}$ of disjoint simple closed curves is jointly primitive if there exist disjoint disks $\left\{D_{1}, \ldots, D_{n}\right\}$ in $H^{\prime}$ such that $\left|a_{i} \cap D_{j}\right|=\delta_{i j}$. This is equivalent to the statement that attaching 2-handles to $H^{\prime}$ along any subset of curves in $\left\{a_{1}, \ldots, a_{n}\right\}$ results in a handlebody Gor87, Wu92a].

Definition 3.3. A surface, $P$, in the boundary of 3-manifold $M$ will be said to be $\partial$-compressible if there is a disk properly embedded in $M$ that intersects $P$ is a single arc which is not parallel into $\partial P$. Otherwise $P$ is $\partial$-incompressible.

Definition 3.4. Let $a, b$, and $c$ be disjoint simple closed curves on genus $g>1$ handlebody $\partial H^{\prime}$ such that $a$ and $b$ are jointly primitive and $a \cup b \cup c$ bounds a pair of pants $P^{\prime} \subseteq \partial H^{\prime}$. It will turn out that such a pair $\left(H^{\prime}, P^{\prime}\right)$ leads to a handlebody containing knots with nontrivial handlebody surgeries, so we say that such a pair is of handlebody type. If furthermore, there is no meridian disk or essential annulus of $H^{\prime}$ disjoint from $c$ (i.e. $c$ is 'disk-busting' and 'annulus-busting', see section 5.1), we say that $\left(H^{\prime}, P^{\prime}\right)$ is of strong handlebody type.

Definition 3.5. Let $a, b$, and $c$ be disjoint simple closed curves on $\partial H^{\prime}$ such that $a$ and $b$ are primitive, $a$ and $b$ are parallel, and $a \cup b \cup c$ bounds a pair of pants $P^{\prime} \subseteq \partial H^{\prime}$ that does not lie in the parallelism between $a$ and $b$. In other words, $P^{\prime}$ is gotten by banding annular neighborhoods of $a$ and $b$ in the complement of the annulus cobounded by $a$ and $b$. A pair $\left(H^{\prime}, P^{\prime}\right)$ satisfying these conditions leads to knots in handlebodies which have surgeries that are the union of 1 -handles with a Seifert fiber space over the disk with two exceptional fibers. So we say that such a pair is of Seifert type. Furthermore, we say $\left(H^{\prime}, P^{\prime}\right)$ is of strong Seifert type if the following two conditions hold: 
- There is no meridian disk or essential annulus of $H^{\prime}$ disjoint from $c$.

- If $H^{\prime}$ has genus 2 , then $P^{\prime}$ is $\partial$-incompressible in $H^{\prime}$.

Lemma 3.6. Let $\left(H^{\prime}, P^{\prime}\right)$ be a genus $g \geq 2$ handlebody and a pair of pants of either handlebody or Seifert type with $\partial P^{\prime}=a \cup b \cup c$ as above. Assume $c$ is disk-busting in $H^{\prime}$. Then $P^{\prime}$ and $\overline{\partial H^{\prime} \backslash P^{\prime}}$ are incompressible in $H^{\prime}$. Furthermore, $P^{\prime}$ and $\overline{\partial H^{\prime} \backslash P^{\prime}}$ are $\partial$-incompressible in $H^{\prime}$ unless $g=2,\left(H^{\prime}, P^{\prime}\right)$ is of Seifert type, and $H^{\prime}=T^{\prime} \times I$ with $c=\partial T^{\prime} \times\{p t\}$ where $T^{\prime}$ is a once-punctured torus. In particular, if c intersects each meridian disk of $H^{\prime}$ at least three times (c is 3-disk-busting) and intersects each essential annulus of $H^{\prime}$ ( $c$ is annulus-busting), then $\left(H^{\prime}, P^{\prime}\right)$ is of strong handlebody or Seifert type.

Proof. Assume $c$ is disk-busting. Clearly $P^{\prime}$ and $\overline{\partial H^{\prime} \backslash P^{\prime}}$ are incompressible in $H^{\prime}$. The $\partial$-incompressibility of $P^{\prime}$ is then equivalent to that of $\overline{\partial H^{\prime} \backslash P^{\prime}}$, so we consider $P^{\prime}$. Assume $D$ is a $\partial$-compressing disk for $P^{\prime}$. Note that $c$ cannot intersect a meridian disk of $H^{\prime}$ just once, since then $c$ would be primitive and hence not disk-busting. Therefore the $\partial$-compressing disk $D$ has to intersect $P^{\prime}$ in a separating arc connecting $c$ to itself. Then a primitivizing disk for $a$ that is disjoint from $D$ can be constructed from another primitivizing disk for $a$ by surgering away outermost intersections with $D$. When $\left(H^{\prime}, P^{\prime}\right)$ is of handlebody type, this can be done with a primitivizing disk for $a$ disjoint from $b$ to produce a new disk that intersects $c$ once - a contradiction. So we assume $\left(H^{\prime}, P^{\prime}\right)$ is of Seifert type. Surger $H^{\prime}$ along $D$. Then $P^{\prime} \backslash D$ is contained in an annulus in the boundary of the resulting genus $g-1$ handlebody, and $a$ and $b$ continue to be primitive. If $g>2$, then we can band a primitivizing disk for $a$ (and $b$ ) to itself to get a meridian disk disjoint from $c$ a contradiction. So we assume $g=2$. Let $T^{\prime}$ be the once-punctured torus in $H^{\prime}$, with boundary $c$, gotten by adding to $P^{\prime}$ the parallelism between $a$ and $b$. Now $a$ is primitive in this solid torus gotten by surgering $H^{\prime}$ along $D$. Cutting again along a meridian disk, $D^{\prime}$, for this solid torus we get the product of a disk and an interval. Regluing along $D, D^{\prime}$ gives the claimed product structure.

The final statement follows immediately from the definition of strong handlebody or Seifert type given the above, once we note that $c$ being 3 -disk-busting guarantees that $P^{\prime}$ is $\partial$-incompressible in $H^{\prime}$.

Definition 3.7. Let $\left(H^{\prime}, P^{\prime}\right)$ be either of handlebody or Seifert type, and $(H, P)$ be as in section 2 Identify $P$ and $P^{\prime}$ so that $\partial_{+} P$ and $\partial_{-} P$ are identified with $a$ and $b$. Since $H$ is a product $P \times I$, the resulting space $M=H \cup H^{\prime}$ is a handlebody containing a properly embedded pair of pants $P$ separating $H$ and $H^{\prime}$. We call $M$ the big handlebody. We refer to $M$ as having (strong) handlebody or (strong) Seifert type according to the constituent $\left(H^{\prime}, P^{\prime}\right)$. As in section 2, let $\tau$ be a simple closed curve in $T$ where $H=T \times I$, and let $K(\tau)$ be the corresponding knot in $H$. Under the above identification of $H$ in $M$ we may then consider $K(\tau), K(\tau(p, q))$ or $K(\tau(\kappa, \alpha, n))$ as the corresponding knot in $M$.

Proposition 3.8. Let $\tau$ be an essential simple closed curve in $T$. If $M$ is of handlebody type, then $K(\tau)$ has a longitudinal handlebody surgery. If $M$ is of Seifert type, then $K(\tau)$ has a longitudinal surgery yielding a $D(p, q)$-Seifert space with $g-1$ attached 1 -handles where $\tau=\tau(p, q)$ and $g$ is the genus of $M$.

Proof. We first show that $K=K(\tau) \subseteq H$ has a handlebody surgery under which $P$ becomes $\partial$-compressible. To see this, recall that $K$ lies in the punctured torus $T \times\{0\}$. Let $S$ be the 3 -punctured sphere $(T \times\{0\}) \cap H_{K}$. This surface defines a slope on $\partial N(K)$, the unoriented isotopy class of any boundary component. Because the geometric intersection number of this slope and the meridian on $\partial N(K)$ is one, we say this slope is longitudinal. Since $K$ is isotopic to a primitive curve on $\partial H$, surgery at this slope yields a genus two handlebody. After surgery, $S$ becomes a separating disk $D$ meeting $P$ in a single essential arc disjoint from $\partial P_{+}$and $\partial P_{-}$(see Figure 3). Call the surgery slope $\gamma$, and denote by $H(\gamma)$ the surgered handlebody.

If $M$ has handlebody type, perform the $\partial$-compression along $D$ and glue the resulting two solid tori to $H^{\prime}$, along annuli whose cores are $a$ and $b$. The cores of the gluing annuli are jointly primitive in $H^{\prime}$, so the result is a handlebody. Reversing the $\partial$-compression does not change this and reconstructs $M$. 
When $M$ has Seifert type, with $\tau=\tau(p, q)$, we need to give coordinates to the $\gamma$ surgery on $P \subseteq H$. As before, $H(\gamma)$ contains a separating disk $D$ meeting $P$ in a single arc. The disk $D$ separates $H(\gamma)$ into two solid tori $J_{+}$and $J_{-}$, with $\partial_{+} P \subseteq J_{+}$and $\partial_{-} P \subseteq J_{-}$.

Recall that as oriented curves $\mu$ and $\lambda$ form an oriented basis of $H_{1}(T)$. Let $H\left[\partial_{-} P\right]$ and $H\left[\partial_{+} P\right]$ be the solid tori gotten by attaching a 2-handle to $H$ along the curves $\partial_{-} P=\mu \times\{-1\}$ and $\partial_{+} P=\lambda \times\{+1\}$ respectively. Thinking of $H \cong T \times I$, we use meridian/longitude coordinates on $H\left[\partial_{-} P\right]$ and $H\left[\partial_{+} P\right]$ given by the pairs of oriented curves $(\mu \times\{1\}, \lambda \times\{1\})$ in the first case and $(\lambda \times\{-1\}, \mu \times\{-1\})$ in the second. (One may care to refer to Figure 1 (Right).) In $H\left[\partial_{-} P\right], K(\tau(p, q))$ becomes a $(p, q)$ curve, with surface slope $\gamma$. Therefore the result of attaching a 2-handle to $H(\gamma)$ along $\partial_{-} P$ is the connect sum of the lens space $L(p, q)$ with a solid torus (cf. [Bow13, Proposition 5.2]). Similarly, the result of attaching a 2-handle to $H(\gamma)$ along $\partial_{+} P$ is the connect sum of the lens space $L(q, p)$ with a solid torus. It follows that $\partial_{-} P$ is a $(p, q)$ curve in $J_{-}$and $\partial_{+} P$ is a $(q, p)$ curve in $J_{+}$.

When $M$ has Seifert type, the curves $\partial_{ \pm} P$ are primitive and isotopic in $\partial H^{\prime}$. Therefore we may think of the space $M(\gamma)$ as the space obtained by identifying an annulus neighborhood of $\partial_{-} P$ in $J_{-}$with an annulus neighborhood of $\partial_{+} P$ in $J_{+}$and attaching $(g-1) 1$-handles. This space is a $D(p, q)$-Seifert space with $g-1$ attached 1-handles, as claimed.

We will need the following technical lemma later:

Lemma 3.9. Let $(H, P)$ be as in section 2, let $\left(H^{\prime}, P^{\prime}\right)$ be of strong handlebody or strong Seifert type, and let $(\widehat{H}, \widehat{P})$ denote either $(H, P)$ or $\left(H^{\prime}, P^{\prime}\right)$. Let $A$ be an annulus properly embedded in $\widehat{H}$ with $\partial A \subset \widehat{P}$. If $\partial A$ bounds an annulus $B \subseteq \widehat{P}$, then $A$ may be isotoped to lie entirely in $\widehat{P}$. If $\partial A$ does not bound an annulus in $\widehat{P}$, then $(\widehat{H}, \widehat{P})=\left(H^{\prime}, P^{\prime}\right)$ of strong Seifert type, and $A$ may be isotoped to the annulus of $H^{\prime} \backslash P^{\prime}$ with boundary $a \cup b$ while keeping $\partial A \subset P^{\prime}$.

Proof. Let $A$ be an annulus properly embedded in $\widehat{H}$ with $\partial A \subseteq \widehat{P}$. First assume $\partial A$ cobounds an annulus $B$ in $\widehat{P}$. The surface $A \cup B$ is a torus $S$. Isotop $S$ slightly inside $\widehat{H}$ to obtain an embedded torus. There is an annulus $C$ so that one boundary component of $C$ lies on $\widehat{P}$ and the other is a core of $B$.

A torus in a handlebody compresses to one side. If $S$ were compressible to the side containing $C$, then using an innermost disk/outermost arc argument we could find a compressing disk $D$ for $S$ not meeting $C$. Therefore we could isotop $D$ so that $\partial D \subseteq \widehat{P}$, contradicting that $\widehat{P}$ is incompressible. So $S$ must be compressible to the side not containing $C$. From the irreducibility of $\widehat{H}$, we conclude that $S$ bounds a solid torus $N$ on this side.

If the core of $B$ is not longitudinal in $N$, then we obtain a reducible manifold after attaching a 2 -handle to $\widehat{H}$ along this curve. When $\widehat{H}=H$, or when $\widehat{H}=H^{\prime}$ and the attaching curve is parallel to one of the primitive components of $\partial P^{\prime}$, it is clear that this does not happen. We wish to show that it does not happen when $\widehat{H}=H^{\prime}$ and the attaching curve is parallel to component $c$ of $\partial P^{\prime}$. Because $c^{\prime}$ meets every meridian disk of $H^{\prime}$, and hence its complement in $\partial H^{\prime}$ is incompressible, the Handle Addition Lemma [CGLS87, Lemma 2.1.1] gives a contradiction.

We have shown that the core of $B$ is longitudinal in $N$, and so we may isotop $A$ to $B$ through $S$ as claimed.

Finally, assume the components of $\partial A$ are not parallel in $\widehat{P}$. Then $A$ cannot lie in $H$ since the boundary components of $P$ represent different homology classes in $H$. So $A$ is in $H^{\prime}$. As there is a meridian disk of $H^{\prime}$ disjoint from $A$, neither component of $\partial A$ can be isotopic to the curve $c$ of $\partial P^{\prime}$ (since this curve is disk-busting). Hence $A$ may be isotoped keeping $\partial A \subset P^{\prime}$ so that $\partial A=a \cup b$. Since $c$ intersects every essential annulus, $A$ must be $\partial$-parallel. Therefore $\left(H^{\prime}, P^{\prime}\right)$ must be of Seifert type and $A$ isotopic to the annulus component of $H^{\prime} \backslash P^{\prime}$.

Assume $M$ is of handlebody or Seifert type. Under the embedding of $H$ in $M$ when $\tau=\tau(\kappa, \alpha, n)$ then the knot $K(\tau)$ in $M$ can be considered, as in Definition 2.6 of section 2, as obtained from the knot $K(\kappa)$ 
by twisting $n$ times along the annulus $\widehat{R}(\alpha)$. As in the preceding section, let $\partial \widehat{R}(\alpha)=L_{+} \cup L_{-}$and $\mathcal{L}(\kappa, \alpha)=K(\kappa) \cup L_{+} \cup L_{-}$in $M$.

Lemma 3.10. Assume $M$ is of strong handlebody or strong Seifert type. Let $\tau=\tau(\kappa, \alpha, n)$. Let $\mathcal{L}=\mathcal{L}(\kappa, \alpha)$. The space $M_{\mathcal{L}}$ is irreducible.

Proof. Because $M$ is of strong handlebody or Seifert type, $P$ is incompressible in $M$. So it suffices to show that $H_{\mathcal{L}}$ is irreducible. Assume $S \subseteq H_{\mathcal{L}}$ is an embedded sphere not bounding a ball. Let $A_{+}, A_{-}, R$ be the restriction to $H_{\mathcal{L}}$ of $A_{+}(\alpha), A_{-}(\alpha), R(\alpha)$ of $H$ (Definition 2.4). If we choose $S$ so that $\left|S \cap\left(A_{+} \cup A_{-} \cup R\right)\right|$ is minimal, an innermost disk argument shows that we may take $S \cap\left(A_{+} \cup A_{-} \cup R\right)=\emptyset$.

The result of cutting $H_{\mathcal{L}}$ along $A_{+} \cup A_{-} \cup R$ is homeomorphic to the space we get by cutting $H$ along the annulus $\alpha \times I$ and removing tubular neighborhoods of a collection of $\operatorname{arcs} a_{i}$ whose ends meet to form $K$. The annulus $\kappa \times[0,1 / 2] \subseteq H_{\mathcal{L}}$ becomes a collection of disks showing that each $\operatorname{arc} a_{i}$ is unknotted, and so the resulting space is a handlebody. The conclusion follows from the fact that handlebodies are irreducible.

Lemma 3.11. Assume $M$ is of strong handlebody or strong Seifert type. Let $\tau=\tau(\kappa, \alpha, n)$. Let $\mathcal{L}=\mathcal{L}(\kappa, \alpha)$. Let $T_{+}, T_{-}$be the components of $\partial M_{\mathcal{L}}$ corresponding to $L_{+}, L_{-}$. There is no essential annulus in $M_{\mathcal{L}}$ with one boundary component on $T_{+}$and the other on $T_{-}$.

Proof. Assume $A$ is such an essential annulus. First we show that we may assume that $A$ lies in $H_{\mathcal{L}}$. Choose $A$ to minimize $|A \cap P|$. Since $A$ and $P$ are incompressible in $M$, there are no simple closed curves of intersection which are trivial in $A$ or $P$. If there are essential simple closed curves of intersection, Lemma 3.9 shows that there are sub-annuli $A_{+}^{\prime}, A_{-}^{\prime}$ of $A$, properly embedded in $H$, that have one boundary component on $T_{+}, T_{-}$(resp.) and the other isotopic to $\partial_{+} P$ or to $\partial_{-} P$, with $\partial A_{+}^{\prime}$ not parallel to $\partial A_{-}^{\prime}$ on $P$. As $L_{+}, L_{-}$ are isotopic in $H$, this says that a power of $\partial_{+} P$ is equal to a power of $\partial_{-} P$ as homotopy classes in $H$. But $\partial_{+} P, \partial_{-} P$ generate the fundamental group of $H$, a contradiction. Hence $A \cap P=\emptyset$.

Thus we take $A$ properly embedded in $H_{\mathcal{L}}$. Let $A_{+}, A_{-}$be the restriction of $A_{+}(\alpha), A_{-}(\alpha)$ to $H_{\mathcal{L}}$. Isotop $\partial A$ and $A_{+} \cup A_{-}$to intersect minimally on $T_{+} \cup T_{-}$. There can be no $\operatorname{arcs}$ of intersection of $A \cap\left(A_{+} \cup A_{-}\right)$ as the signs of intersections are consistent along each boundary component of $H_{\mathcal{L}}$. (In particular, $\partial A$ must be disjoint from $A_{+} \cup A_{-}$.)

Suppose then that $A$ meets $A_{+} \cup A_{-}$minimally. We may choose a simple closed curve of intersection $\gamma$ which is outermost in $A_{+} \cup A_{-}$in the sense that it bounds an annulus $A^{\prime} \subset A_{+} \cup A_{-}$with one boundary component on $T_{+} \cup T_{-}$so that int $A^{\prime} \cap A=\emptyset$. By cutting and pasting along $\gamma$ we get a new annulus with one boundary component on $T_{i}$ and one on $T_{j}$ which meets $A_{+} \cup A_{-}$fewer times. This is impossible by minimality of $\left|A \cap\left(A_{+} \cup A_{-}\right)\right|$, so we may assume that $A \cap\left(A_{+} \cup A_{-}\right)=\emptyset$.

The surface $A_{+} \cup A \cup A_{-}$in $H_{\mathcal{L}}$ then extends to an annulus $B$ in $H$ with $\partial B=\alpha \times\{-1,+1\}$ and $B \cap K(\kappa)=\emptyset$. Then, carrying along $K(\kappa)$, we may isotop $B$ in $H$ to be vertical with respect to the product structure $T \times I$ of $H$. The image of $B$ under the projection $H=T \times I \rightarrow T$ is the curve $\alpha$, and the image of $K(\kappa)$ is homotopic to $\kappa$. Since $B$ is disjoint from $K(\kappa)$, the algebraic intersection number of $\alpha$ and $\kappa$ in $T$ should be zero. But by definition, $\alpha$ and $\kappa$ are not isotopic in $T$, a contradiction.

Proposition 3.12. Assume $M$ is of strong handlebody or strong Seifert type. Let $\tau$ be an essential curve in $T$ and consider $K(\tau)$ in $M$.

- The space $M_{K(\tau)}$ is irreducible and atoroidal.

- When $\tau \notin\{\lambda, \mu, \nu\}, M_{K(\tau)}$ is $\partial$-irreducible.

- When $\tau \notin\{\lambda, \mu, \nu, \lambda-\mu, \lambda+\nu, \mu+\nu\}$, there is no essential annulus in $M_{K(\tau)}$. 
Proof. The fact that $M_{K(\tau)}$ is irreducible is implied by the irreducibility of $H_{K(\tau)}$ because $P$ is incompressible in $H$ and $H^{\prime}$. The space $H_{K(\tau)}$ is irreducible since there are meridian disks of $H$ that have nonzero algebraic intersection with $K(\tau)$ by our hypothesis that $\tau$ is an essential curve in $T$.

Suppose that $S \subseteq M_{K(\tau)}$ is an essential torus chosen to minimize $|S \cap P|$. If $|S \cap P|>0$, these simple closed curves must be essential in both $S$ and $P$ (because both $S$ and $P$ are incompressible). By Lemma 3.9 then, each component of $S \cap H$ is an annulus parallel into $P$. By the same Lemma, and the minimality of intersection, we conclude that $\left(H^{\prime}, P^{\prime}\right)$ has Seifert type and each component of $S \cap H^{\prime}$ is an annulus whose boundary is a pair of curves that are not parallel in $P$. Thus there must be annular components of $S \cap H$ parallel into disjoint annuli in $P$. One of these parallelisms must be disjoint from $K(\tau)-$ contradicting the minimality of $|S \cap P|$. Thus $S \cap P=\emptyset$. Since the handlebody $H^{\prime}$ and compression body $H_{K(\tau)}$ are atoroidal, no such essential torus can exist.

Assume $\tau \notin\{\lambda, \mu, \nu\}$. Suppose that $D$ is a $\partial$-reducing disk for $M_{K(\tau)}$ chosen to minimize $|D \cap P|$. An arc of intersection $D \cap P$, outermost in $D$, cuts off a subdisk $D^{\prime} \subseteq D$ which is a $\partial$-compressing disk for $P \subseteq M$. There are no such disks in $H^{\prime}$ since $\left(H^{\prime}, P^{\prime}\right)$ is of strong handlebody or Seifert type. By Lemma 2.11 if there is such disk in $H$ then $\tau=\lambda$, $\mu$, or $\nu$ contrary to hypothesis. Hence $D \cap P=\emptyset$. But $P, \partial H \backslash P$ are incompressible in $H$ by Lemma 2.11. and $P^{\prime}, \partial H^{\prime} \backslash P^{\prime}$ are incompressible in $H^{\prime}$ since $\left(H^{\prime}, P^{\prime}\right)$ is a strong type.

Finally, assume $\tau \notin\{\lambda, \mu, \nu, \lambda-\mu, \lambda+\nu, \mu+\nu\}$. Suppose that $A$ is an essential annulus properly embedded in $M_{K(\tau)}$, chosen to minimize $|A \cap P|$. Because $M_{K(\tau)}$ contains no essential tori, we cannot have $\partial A \subseteq$ $\partial N(K(\tau))$. So suppose that one component of $\partial A$ lies in $\partial N(K(\tau))$ and the other lies in $\partial M$. By the minimality of $|A \cap P|$, we may assume that there are no arcs of intersection. Therefore there is a subannulus $A^{\prime} \subseteq A$ with one boundary component on $\partial N(K(\tau))$ and the other on $\partial H$, either in $P$ or $\partial H \backslash P$. This annulus shows that a power of $K(\tau)$ is homotopic to a component of $\partial P$. Since both $K(\tau)$ and each component of $\partial P$ is primitive, no component of $\partial P$ is homotopic to a proper power of $K(\tau)$. On the other hand, $K(\tau)$ is not homotopic to a component of $\partial P$ since $\tau \neq \lambda, \mu$ or $\nu$.

Suppose then that $\partial A \subseteq M$. By minimality of $|A \cap P|$, there can be no arcs of intersection which are trivial in either $A$ or $P$ (Lemma 2.11 and $\partial$-irreducibility). If there are nontrivial arcs of intersection, we may choose two which bound a disk in $A$ whose interior lies in $H$. With our restriction on $\tau$, this contradicts Lemma 2.13. If there are simple closed curves of intersection, there is a subannulus $A^{\prime} \subseteq A$ lying entirely in $H$. We may isotop $A^{\prime}$ so that both components of $\partial A^{\prime}$ lie in $P$. By Lemma 3.9 and the restriction on $\tau$, this annulus must be boundary parallel into $P$. Hence $A$ may be isotoped to further reduce $|A \cap P|$ contrary to the minimality. Thus $A$ is disjoint from $P$ and lies entirely in $H$ or $H^{\prime}$. Again, Lemma 3.9 (and the restriction on $\tau$ in case $A \subset H$ ) or the fact that $c$ is annulus-busting ( $P^{\prime}$ is of strong type) implies that $A$ is not essential.

\section{Essential surfaces}

In section 5, we will study a family of knots $K_{i}$ in a handlebody $M$ obtained by twisting a knot $K=K_{0}$ along an annulus $\widehat{U}$ with one boundary component $\gamma$ in $\partial M$ and the other a knot $L$ in the interior of $M$. We want to show that, generically, these knots are distinct and hyperbolic. The difference in knot type will come from showing that the minimum number of times $K_{i}$ intersects a meridian disk of $M$ (the "disk hitting number") increases with $|i|$, Proposition 5.18(4). The hyperbolicity will come from showing that the exteriors of these knots are irreducible, atoroidal, $\partial$-irreducible, and anannular, Proposition 5.19 by way of Lemma 5.17 and Proposition $5.18(5)$.

In this section, we establish Theorem 4.2 below which is applied in Proposition 5.18 to show that for large twisting numbers $|i|, K_{i}$ must intersect any meridian disk or essential annulus of $M$ many times. In application, we find a catching surface $Q$ and use it to generate a lower bound on the intersection number 
of $K_{i}$ with an essential surface (such as a meridian disk or essential annulus) in $M$ which restricts to a surface $F$ properly embedded in the exterior of $K_{i}$ and $L$. As the pair $\left(M, K_{i}\right)$ is homeomorphic to the pair $\left(M_{L}(-1 / i), K\right)$, where $M_{L}(-1 / i)$ is the manifold obtained by doing $-1 / i$-surgery on $L$ in $M$, we may view $F$ as a surface in the exterior of $K \cup L$ in $M$ whose boundary components have framing $-1 / i$ on $L$. The catching surface will be chosen so that its boundary has framing on $L$ whose intersection number on $\partial N(L)$ with $\partial F$ increases with $|i|$ (that is, $\Delta_{L}$ below increases with $|i|$ ).

Theorem 4.2 however is more general. In particular, it does not require that we be in the context of twisting along an annulus $\widehat{U}$. The argument mimics that of Lemma 2.8 of [BGL13]. It shows that, for an essential surface $F$ and a catching surface $Q$ in $M_{K \cup L}$, if the boundary slopes of the surfaces $F$ and $Q$ along the knot $L$ intersect more than a certain measure of complexity of $F$ and $Q$ then there must be certain kinds of annuli or Möbius bands in $M_{K \cup L}$.

The remainder of this section is devoted to the proof of Theorem 4.2. We first establish the necessary notation.

Let $M$ be a compact, connected oriented 3-manifold with knots $K$ and $L$. Let $X$ be the exterior of $K$ and $L$ in $M$. Let $T_{K}, T_{L}$ be the torus components of $\partial X$ corresponding to $K, L$. Note that the rest of the boundary of $X$ is the boundary of $M$, i.e. $\partial X-\left(T_{K} \cup T_{L}\right)=\partial M$.

Assume $F$ is a compact, connected, orientable surface properly embedded in $X$. For $j=K, L$ let $f_{j}=$ $\left|\partial F \cap T_{j}\right|$ and let $\alpha_{j}$ be the slope of the curves $\partial F \cap T_{j}$ in $T_{j}$. Also let $f_{M}=|\partial F \cap \partial M|$. We assume throughout this section that $f_{L} \geq 1$. Define $f_{K}^{\prime}=\max \left(f_{K}, 1\right)$ and $f_{M}^{\prime}=\max \left(f_{M}, 1\right)$.

Definition 4.1 (Catching surface and associated distances). Let $Q$ be an oriented surface with $\partial Q \cap T_{L}$ being a non-empty set of coherently oriented curves in $T_{L}$ (with orientation from $Q$ ). We also let $\beta_{j}$ denote the slope of the curves $\partial Q \cap T_{j}$ for $j=K, L$. Then we define $\Delta_{j}$ to be $\Delta\left(\alpha_{j}, \beta_{j}\right)$ on $T_{j}$ for $j=K, L$ (see Definition 2.9, where $\Delta_{K}=0$ when $f_{K}=0$. Also set $\Delta_{K}^{\prime}=\max \left(\Delta_{K}, 1\right)$. Similarly define $\Delta_{M}$ to be the maximum geometric intersection number between a component of $\partial Q \cap \partial M$ and a component of $\partial F \cap \partial M$; we also set $\Delta_{M}=0$ when $f_{M}=0$. If $\Delta_{L}>0$ then $Q$ is called a catching surface for $(F, K, L)$.

Theorem 4.2. Let $K$ and $L$ be knots in $M$ and $X$ be their exterior, with $T_{L}$ the component of $\partial X$ corresponding to $L$. Let $F$ be a compact, connected, orientable, properly embedded surface in $X$ which is boundary incompressible at $T_{L}$ (and $\left|F \cap T_{L}\right| \neq \emptyset$ ), and let $Q$ be a catching surface for $(F, K, L)$ (Definition 4.1). If

$$
\Delta_{L}>6 f_{M}^{\prime} \max (-6 \chi(Q), 2)\left(f_{K}^{\prime} \Delta_{K}^{\prime}+f_{M}-\chi(\widehat{F})+2\right)
$$

then one of the following is true:

1. There exists a Mobius band properly embedded in $X$ with boundary isotopic to $\alpha_{L}$ (the slope of $\partial F$ ) in $T_{L}$.

2. There exists an annulus properly embedded in $X$ whose boundary is an essential curve in $T_{L}$ and a curve in $\partial M$.

Remark 4.3. Note that if $F$ is incompressible, then it is automatically $\partial$-incompressible at $L$ unless $F$ is an annulus parallel into $T_{L}$.

Remark 4.4. Theorem 4.2 holds more generally when $K$ is a link of multiple components. Just let $T_{K}$ be the union of the corresponding torus components of $\partial X$ and define $\Delta_{K}$ to be the maximum of $\Delta(\alpha, \beta)$ for slopes $\alpha$ of $\partial F$ and $\beta$ of $\partial Q$ among components of $T_{K}$, and then $\Delta_{K}^{\prime}=\max \left(\Delta_{K}, 1\right)$. The proof below carries through unchanged.

Proof. Let $F$ and $Q$ be as above, and take $j \in\{K, L\}$. Fixing an orientation on $F(Q)$, we call two components of $\partial F \cap T_{j}\left(\partial Q \cap T_{j}\right.$, resp.) parallel if they inherit coherent orientations on $T_{j}$. They are antiparallel otherwise. For example, by definition, all components of $\partial Q \cap T_{L}$ are parallel. Label the components 

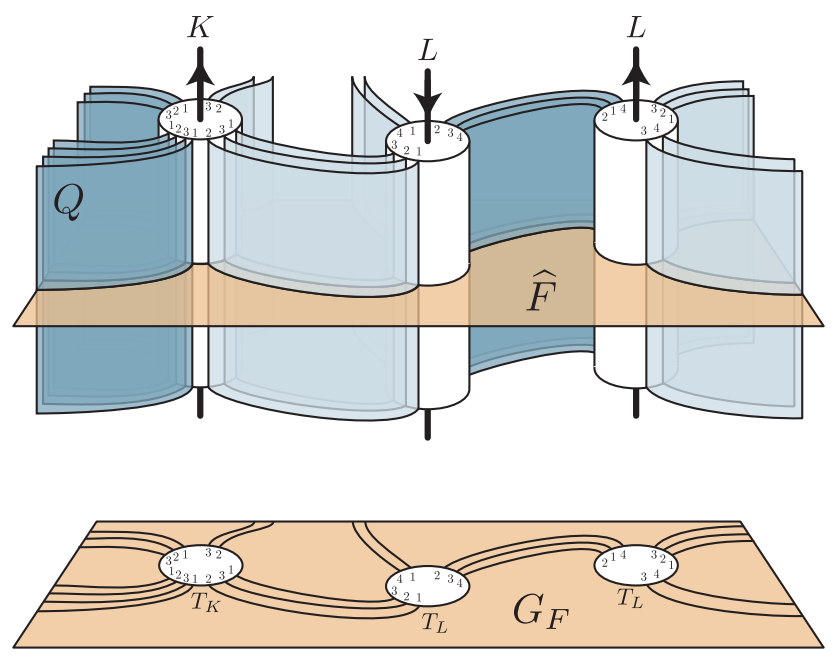

Figure 5: An example of a portion of the labelled graph $G_{F}$ arising from the intersection of $Q$ with $F$ and, say, $\left|\partial Q \cap T_{K}\right|=3$ with $\Delta_{K}=4$ and $\left|\partial Q \cap T_{L}\right|=4$ with $\Delta_{L}=2$. Labels of $G_{F}$ are given by the corresponding component of $\partial Q \cap \partial X$.

of $\partial F \cap T_{j}$ from 1 to $\left|\partial F \cap T_{j}\right|$ in sequence along $T_{j}$. Similarly label the components of $\partial Q \cap T_{j}$. Label the components of $\partial F \cap \partial M(\partial Q \cap \partial M)$ arbitrarily. Assume that $F$ and $Q$ have been isotoped to intersect transversally and minimally. Abstractly cap off the boundary components of these surfaces with disks to form the respective closed surfaces $\widehat{F}$ and $\widehat{Q}$. (Note that $\chi(\widehat{F})=\chi(F)+f_{K}+f_{L}+f_{M}$.) Regarding these capping disks as fat vertices and the arcs of the intersection $F \cap Q$ as edges we create the fat-vertexed graphs $G_{F}$ and $G_{Q}$ in the respective closed surfaces $\widehat{F}$ and $\widehat{Q}$. Label the endpoint of an edge in one graph with the vertex of the other graph whose boundary contains the endpoint.

Figure 5 gives an example of how the labelled graphs $G_{Q}, G_{F}$ arise.

Two vertices on $G_{F}\left(G_{Q}\right)$ are parallel or anti-parallel if the corresponding boundary components of $F(Q$, resp.) are parallel or anti-parallel (in particular, for vertices to be parallel or anti-parallel they must corresond to boundary components which are both on $T_{K}$ or both on $T_{L}$ ). The orientability of $F, Q$, and $X$ give the

Parity Rule: An edge connecting parallel vertices on one graph must connect anti-parallel vertices on the other graph.

Observe that a vertex of $G_{Q}$ corresponding to $T_{j}$ has valence $f_{j} \Delta_{j}$. Let $V_{j}$ be the set of vertices in $G_{Q}$ that corresponds to $T_{j}$ for $j=K, L$, and let $V_{M}$ be the set of vertices corresponding to $\partial M$. Recall that because $Q$ is catching, $V_{L}$ is non-empty. Since $F$ is $\partial$-incompressible along $T_{L}$, the Parity Rule guarantees that $G_{Q}$ contains no monogons (i.e. 1-sided faces) at any vertex of $V_{L}$.

Assume

$$
\frac{\Delta_{L}}{\max (-6 \chi(Q), 2)}>6 f_{M}^{\prime}\left(f_{K}^{\prime} \Delta_{K}^{\prime}+f_{M}-\chi(\widehat{F})+2\right)>0
$$

and note that the second inequality does indeed hold true since $f_{K}^{\prime} \Delta_{K}^{\prime} \geq 1$.

An edge of a graph is called trivial if it bounds a monogon face. Two edges are parallel if there is a sequence of bigon faces between them.

Claim 4.5 (cf. Claim 2.9 BGL13]). The graph $G_{Q}$ contains at most $\max (-3 \chi(Q), 1)$ parallelism classes of non-trivial edges. 
Proof. Pick one edge from each parallelism class of non-trivial edges in $G_{Q}$ and consider it as an arc properly embedded in the surface $Q$. Let $E$ be the collection of all such arcs. Then $|E|$ is the number of parallelism classes in $G_{Q}$. First note that $|E| \leq 1$ when $\chi(Q) \geq 0$. So we assume $\chi(Q)<0$. Because the edges in $E$ are not boundary parallel in $Q$, they can be completed to an ideal triangulation of (the interior of) $Q$ by adding more arcs between the components of $\partial Q$ as needed. If $E^{\prime}$ is this resulting number of edges and $F$ is the number of ideal triangles, then we have both $3 F=2 E^{\prime}$ and $\chi(Q)=-E^{\prime}+F$. Thus $E \leq E^{\prime}=-3 \chi(Q)$.

Since any vertex of $V_{L}$ has valence $f_{L} \Delta_{L}$ (and there are no monogons of $G_{Q}$ at vertices of $V_{L}$ ), Claim 4.5 shows that there exists a set $\mathcal{E}$ of at least $\left\lceil\frac{\left(f_{L} \Delta_{L} / 2\right)}{\max (-3 \chi(Q), 1)}\right\rceil=\left\lceil\frac{f_{L} \Delta_{L}}{\max (-6 \chi(Q), 2)}\right\rceil$ mutually parallel edges in $G_{Q}$ with an end point at a vertex of $V_{L}$, where $\lceil x\rceil$ is the smallest integer not less than $x$. There are three cases for the other end point of these edges: either it is at a vertex of (a) $V_{L}$ (perhaps the same vertex); (b) $V_{M}$; or (c) $V_{K}$. By the assumed inequality $(*)$ above, $|\mathcal{E}| \geq \frac{f_{L} \Delta_{L}}{\max (-6 \chi(Q), 2)}>f_{K} \Delta_{K}$, so there are more edges in $\mathcal{E}$ than the valence of any vertex in $V_{K}$; hence the other end point of these edges cannot be in $V_{K}$ and $(c)$ does not arise. Also note that the inequality $(*)$ implies that $\mathcal{E}$ contains at least two edges, more than $f_{L}$ edges, and more than $f_{M}$ edges.

Let $\widehat{F}_{L}$ be the subsurface of $\widehat{F}$ obtained by capping off the boundary components of $F$ in $T_{L}$ and $\widehat{F}_{L, M}$ be obtained by further capping off any boundary components of $\widehat{F}_{L}$ in $\partial M$.

For case (a), form the subgraph $G_{F_{L}}(\mathcal{E})$ of $G_{F}$ in the surface $\widehat{F}_{L}$ that consists of the edges $\mathcal{E}$ and all the $f_{L}$ vertices of $\partial F \cap T_{L}$.

For case (b), form the subgraph $G_{F_{L, M}}(\mathcal{E})$ of $G_{F}$ in the surface $\widehat{F}_{L, M}$ that consists of the edges $\mathcal{E}$ and all the $f_{L}$ vertices of $\partial F \cap T_{L}$ and all $f_{M}$ vertices of $\partial F \cap \partial M$.

Now we have

$$
\begin{aligned}
|\mathcal{E}| & \geq \frac{f_{L} \Delta_{L}}{\max (-6 \chi(Q), 2)} \\
& \geq 6 f_{L} f_{M}^{\prime}\left(f_{K}^{\prime} \Delta_{K}^{\prime}+f_{M}-\chi(\widehat{F})+2\right) \\
& \geq 6 f_{L} f_{M}^{\prime}\left(f_{K}^{\prime}+f_{M}-\chi(\widehat{F})+1\right)+6 f_{L} f_{M}^{\prime}
\end{aligned}
$$

In particular, we have for case (a)

$$
|\mathcal{E}| \geq 3 f_{L}\left[\max \left(1-\chi\left(\widehat{F}_{L}\right), 0\right)\right]+3 f_{L}
$$

Using that $2 f_{L} f_{M}^{\prime}=2 f_{L} f_{M} \geq\left(f_{L}+f_{M}\right)$ in case (b), we get

$$
|\mathcal{E}| \geq 3\left(f_{L}+f_{M}\right)\left(f_{M}+1-\chi\left(\widehat{F}_{L, M}\right)\right)+3\left(f_{L}+f_{M}\right)
$$

Observe that neither $G_{F_{L}}(\mathcal{E})$ nor $G_{F_{L}, M}(\mathcal{E})$ has any monogons. In the former, case (a), this is because the vertices of $V_{L}$ are parallel and the Parity Rule. In the latter, case (b), this is because the edges connect vertices corresponding to different components of $\partial X$.

Claim 4.6 (cf. Claim 2.10 [BGL13]). Let $G$ be a graph in a surface $S$. Let $V$ be the number of vertices and $E$ the number of edges of $G$, and let $\chi(S)$ be the Euler characteristic of $S$. If $G$ has no monogons and $E>3 V \max (1-\chi(S), 1)$, then $G$ has parallel edges.

Further assume either $\chi(S)>0$ or $\partial S \neq \emptyset$. If $G$ has no monogons and $E \geq 3 V \max (1-\chi(S), 1)$, then $G$ has parallel edges. That is, equality also guarantees parallel edges in these cases.

Proof. Assume there are no parallel edges in $G$, and let $V, E$ be the number of vertices, edges. Assume $E>3 V \max (1-\chi(S), 1)$. Then we may add edges to $G$ so that all faces are either $m$-gons with $m \geq 3$ 
or annuli with one boundary component being a component of $\partial S$ and the other consisting of a single edge and vertex of $G$. Since this doesn't change the number of vertices and only increases the number of edges, we still have $E>3 V \max (1-\chi(S), 1)$. Now $\chi(S)=V-E+F$ where $F$ is the number of disk faces of $G$. Because every edge of $G$ is on the boundary of the faces (including the annular faces) twice, $2 E \geq 3 F+|\partial S|$. Let $C=3 \max (1-\chi(S), 1) \geq 3$. Our assumption that $E>C V$ shows both that $V<E / C$ and $E>C$.

Therefore $\chi(S)=V-E+F<E / C-E+2 E / 3-|\partial S| / 3$. Hence $C \chi(S)<E(1-C / 3)-|\partial S| C / 3$. Since $C \geq 3$, this implies that $\chi(S)<0$. Then $C=3(1-\chi(S))$. So $C \chi(S)<E(\chi(S))-|\partial S|(1-\chi(S))$. Consequently, $C>E+(1-1 / \chi(S))|\partial S| \geq E$. This contradicts that $C<E$.

Now assume either $\chi(S)>0$ or $\partial S \neq \emptyset$. Change the strict inequalities $<$ and $>$ above to $\leq$ and $\geq$. Then $C \chi(S) \leq E(1-C / 3)-|\partial S| C / 3$ implies that $\chi(S) \leq 0$, hence $|\partial S|>0$. A second application of the preceding inequality says that in fact $\chi(S)<0$. As above we conclude that $C \geq E+(1-1 / \chi(S))|\partial S|>E$, contradicting that $C \leq E$.

Applying Claim 4.6. $G_{F_{L}}(\mathcal{E})$ in case (a) has parallel edges (with $S=\widehat{F}_{L}, V=f_{L}, E=|\mathcal{E}|$, using (**)), and $G_{F_{L, M}}(\mathcal{E})$ in case (b) has parallel edges (with $S=\widehat{F}_{L, M}, V=f_{L}+f_{M}, E=|\mathcal{E}|$, using $(* * *)$ and $f_{M}>0$ ). That is, there exist edges $e, e^{\prime} \in \mathcal{E}$ bounding rectangles $D_{Q}$ in $G_{Q}$ and $D_{F}$ in $G_{F_{L}}(\mathcal{E})$ or $G_{F_{L, M}}(\mathcal{E})$ such that $D_{Q} \cap D_{F}=\left\{e \cup e^{\prime}\right\}$ (after possibly surgering away simple closed curves of intersection in the interiors of these disks).

Remark 4.7. Note that Claim 4.6 allows for vertices with valence 0 , which may occur in its application to $G_{F_{L, M}}$, at vertices corresponding to $\partial M$.

In case (a), $D_{Q} \cup D_{F}$ is a Möbius band in $X$ with boundary on $T_{L}$ of slope $\alpha_{L}$. This follows from the proof of Lemma 2.1 of Gor98. Following that proof, the boundary of the Möbius band has the slope of $\partial F$ since the rectangle $D_{F}$ connects anti-parallel vertices in $F\left(D_{Q}\right.$ connects parallel vertices in $Q$, hence the Parity Rule guarantees that $D_{F}$ connects anti-parallel vertices). This is conclusion (1) of the Theorem.

In case (b), $D_{Q} \cup D_{F}$ is an annulus in $X$ with a boundary component on each of $T_{L}$ and $\partial M$. The boundary component of this annulus must intersect a component of $\partial F$ and of $\partial Q$ algebraically a non-zero number of times on $T_{L}$. Thus the boundary component of this annulus is essential on $T_{L}$ (and isotopic to neither a component of $\partial F$ nor $\partial Q$ ). This is conclusion (2) of the Theorem.

\section{Constructing $M$ of strong handlebody or Seifert type}

\subsection{Disk-busting and annulus-busting curves}

We show how to construct disk-busting and annulus-busting curves in the boundary of a handlebody.

Definition 5.1. Let $M$ be an orientable 3-manifold and $\gamma$ a collection of simple closed curves in $\partial M$. We say that $\gamma$ is $n$-disk-busting for a positive integer $n$ if any properly embedded disk in $M$ that intersects $\gamma$ fewer than $n$ times is boundary parallel. We simply say $\gamma$ is disk-busting if it is 1-disk-busting. We say $\gamma$ is annulus-busting in $M$ if it is disk-busting and if any annulus properly embedded in $M$ and disjoint from $\gamma$ is compressible or boundary parallel in $M$.

In the discussion below, we then focus on essential disks and annuli in $M$.

Definition 5.2. A disk properly embedded in a 3-manifold $M$ is called essential if it is not parallel into the boundary of $M$. An annulus properly embedded in $M$ is said to be essential if it is incompressible and not parallel into the boundary of $M$.

Lemma 5.3. Let $\gamma$ be a collection of curves in a component of $\partial M$ of genus $g$. If $\gamma$ is 1-disk-busting, then either $g=1$ or $\gamma$ is 2-disk-busting. 
Proof. Assume there is a disk $D$ in $M$ that $\gamma$ intersects once. Then using a component of $\gamma$ to band two copies of $D$ to itself gives an essential disk disjoint from $\gamma$. But $\gamma$ is disk-busting.

Definition 5.4. Recall that a pair of pants is a surface homeomorphic to a 2-sphere minus three disjoint open disks. For a pair of pants $P$, a seam is an essential properly embedded arc in $P$ with endpoints on distinct components of $\partial P$. Observe that a pair of pants $P$ has three isotopy classes of seams and they have mutually disjoint representatives.

Definition 5.5. Let $\Sigma$ be a connected, closed, compact surface of genus at least two. A pants decomposition of $\Sigma$ is a collection $\mathcal{P}$ of simple closed curves in the surface such that the complement of the curves in the surface is a collection of (interiors of) pairs of pants.

- Given a pants decomposition $\mathcal{P}$ of $\Sigma$, a collection of curves $\gamma$ embedded in $\Sigma$ is called $k$-seamed with respect to $\mathcal{P}$ for an integer $k \geq 0$ if, for each pair of pants $P \in \Sigma-\mathcal{P}$, the intersection $\gamma \cap P$ is a collection of seams with at least $k$ members in each isotopy class.

- For a handlebody $H$, a pants decomposition $\mathcal{P}$ of $\partial H$ is compatible with $H$ if every component of $\mathcal{P}$ bounds a disk in $H$.

Lemma 5.6 (Cf. Lemma 2.10, Yos14). Let $H$ be a handlebody and let $\mathcal{P}$ be a pants decomposition of $\partial H$ that is compatible with $H$. If a collection of curves $\gamma$ in $\partial H$ is $k$-seamed with respect to $\mathcal{P}$, then $\gamma$ is $k$-disk-busting in $H$.

Proof. Let $D$ be an essential disk of $H$ and isotope it so that $|\partial D \cap \gamma|$ is minimal. Under this restriction, isotope $D$ to intersect $\mathcal{P}$ minimally. If $D$ is disjoint from $\mathcal{P}$, then clearly the result holds. Let $\mathcal{D}(\mathcal{P})$ be the collection of disks in $H$ bounded by $\mathcal{P}$. We may assume that there are no simple closed curves of intersection between $D$ and $\mathcal{D}(\mathcal{P})$. An arc of intersection $c$ that is outermost on $D$ cuts off a disk $\Delta$ whose boundary is $c \cup e$ where $e \subset \partial D$ is properly embedded in a pair pants coming from $\mathcal{P}$. The arc $e$ cannot be parallel into $\mathcal{P}$, else we could isotope $D$ to reduce its intersection with $\gamma$ or $\mathcal{P}$. Thus $e$ is an essential separating arc in this pair of pants and hence must intersect at least $k$ seams.

Definition 5.7. Let $\gamma_{i}$ be a collection of simple closed curves in the boundary of the 3-manifold $M_{i}$, $i \in\{1,2\}$. Let $b_{i}$ be a band in $\partial M_{i}$ running from $\gamma_{i}$ to itself. That is, $b_{i}=[0,1] \times[0,1]$ is embedded in $\partial M_{i}$ such that $\gamma_{i} \cap b_{i}=\{0,1\} \times[0,1]$. The boundary plumbing of the pairs $\left(M_{1}, \gamma_{1}\right)$ and $\left(M_{2}, \gamma_{2}\right)$ along $b_{1}, b_{2}$ is the pair $(M, \gamma)$ where

- the 3-manifold $M$ is the gluing of $M_{1}$ and $M_{2}$ via a homeomorphism $h: b_{1} \rightarrow b_{2}$ that identifies $\{0,1\} \times[0,1]$ of $b_{1}$ with $[0,1] \times\{0,1\}$ of $b_{2} ;$ and

- the collection of curves $\gamma$ in $\partial M$ is the closure of $\left(\gamma_{1}-b_{1}\right) \cup\left(\gamma_{2}-b_{2}\right)$.

In the plumbing construction, we refer to $b_{i}$ as a plumbing band, and say it is non-trivial if the core of the band, $[0,1] \times\{1 / 2\}$, is not isotopic rel boundary into $\gamma_{i}$.

Note that $b_{1}=b_{2}$ becomes a properly embedded disk, $D$, in $M$ which intersects $\gamma$ four times. We refer to $D$ as the decomposing disk for the boundary plumbing $(M, \gamma)$.

Lemma 5.8. Let $\gamma_{i}$ be a collection of essential simple closed curves in $\partial M_{i}$ which are 3-disk-busting in $M_{i}, i \in\{1,2\}$. Let $(M, \gamma)$ be a boundary plumbing of $\left(M_{1}, \gamma_{1}\right)$ with $\left(M_{2}, \gamma_{2}\right)$ where the plumbing bands are non-trivial. Then

1. No component of $\gamma$ is trivial and $\gamma$ is 3-disk-busting in $M$.

2. If $A$ is a properly embedded annulus in $M$ disjoint from $\gamma$ and which is neither compressible nor boundary parallel in $M$, then $A$ can be isotoped in $M-\gamma$ so that it is disjoint from $\gamma \cup D$ where $D$ is the decomposing disk of the boundary plumbing. 


\section{If each $\gamma_{i}$ is annulus-busting in $M_{i}$, then $\gamma$ is annulus-busting in $M$.}

Proof. Let $D$ be the decomposing disk of the boundary plumbing. To see that no component of $\gamma$ is trivial we note that any such would have to intersect $D$. Let $E$ be the disk bounded by an innermost trivial component. Then an arc of $\partial D \cap E$ that is outermost on $D$ shows that either a component of $\gamma_{i}$ or the plumbing band of $\gamma_{i}$ is trivial in $\partial M_{i}$.

Note that the assumption that no component of $\gamma_{i}$ is trivial implies that the components of $\partial M_{i}$ in which they lie cannot be 2 -spheres.

First we prove that $\gamma$ is 3-disk-busting. Assume not, that there is a essential disk $E$ in $M$ that intersects $\gamma$ minimally and at most twice. We take $E$ to intersect $D$ minimally among such disks. Then it has no simple closed curves of intersection with $D$ (an innermost such on $E$ must be boundary parallel in some $M_{i}$ ) and any arc of intersection must separate the four points $\gamma \cap D$ in $\partial D$. It cannot be disjoint from $D$ else, since $\gamma_{i}$ is 3-disk-busting, $\partial E$ and $\partial D$ would have to cobound an annulus in some $\partial M_{i}$, contradicting that the band of the plumbing is non-trivial. Consider an $\operatorname{arc} \tau$ of $E \cap D$ that is outermost in $E$ and let $\delta$ be the outermost disk that it bounds. We may assume that $\delta$ lies in $M_{1}$. Then $\delta$ cannot be disjoint from $\gamma$, else surgering $D$ along $\delta$ would give a disk in $M_{1}$ intersecting $\gamma_{1}$ at most twice. Such a disk would be trivial in $M_{1}$ and imply that either the plumbing band or some component of $\gamma_{1}$ is trivial. Thus we may assume $\delta$ intersects $\gamma$ once. Note that this means that $E$ intersects $\gamma$ twice, all the $\operatorname{arcs}$ of $E \cap D$ separate these points of intersection on $\partial E$, and besides $\tau$ there is exactly one other outermost arc, $\tau^{\prime}$. We can view $D$ as a rectangle with corners on $\gamma$ and with one pair of opposing sides in $\gamma_{1}$ and the other in $\gamma_{2}$. Using $\delta$ to surger $D$ we again get a contradiction (e.g. to the minimality of intersection with $E$ ), unless $\tau$, viewed in this rectangle $D$, intersects $\gamma_{1}$ twice and is disjoint from $\gamma_{2}$. Letting $\delta^{\prime}$ be the subdisk of $E$ bounded by $\tau^{\prime}$, the same argument applied to $\delta^{\prime}$, shows that $\tau^{\prime}$ is disjoint from $\gamma_{2}$ and that $\delta^{\prime}$ also lies in $M_{1}$. Let $\delta^{\prime \prime}$ be the disk of $E-D$ contiguous to $\delta$. Then $\delta^{\prime \prime}$ lies in $M_{2}$ and intersects $\gamma_{2}$ at most once. Thus $\delta^{\prime \prime}$ is boundary parallel in $M_{2}$. But this contradicts that each component of $\gamma_{2}$ is essential or the minimality of $E \cap D$. Thus we conclude that $\gamma$ is 3-disk-busting in $M$.

To prove part (2), assume $A$ is a properly embedded annulus in $M$ which is disjoint from $\gamma$ and neither compressible nor boundary parallel. Isotop $A$ to intersect $D$ minimally and assume that $A$ is not disjoint from $D$. There are no simple closed curves of intersection (each such would have to bound a disk in $A$ and an innermost such on $A$ will have to give a boundary parallel disk in some $\left.M_{i}\right)$. Furthermore, an $\operatorname{arc}$ of $A \cap D$ must run between different boundary components of $A$, else surgering $D$ along an outermost disk in $A$ gives, as argued above, a trivial disk in some $M_{i}$ which would imply that some component of $\gamma_{i}$ or the plumbing band in $M_{i}$ is trivial. Let $\tau$ be an arc of $E \cap D$ that is outermost on $D$ and let $\delta$ be the corresponding outermost subdisk of $D$. Then $\partial$-compressing $A$ along $\delta$ gives a disk which is disjoint from $A$ and which intersects $\gamma$ at most twice. Since $\gamma$ is 3 -disk-busting, this disk must be boundary parallel in $M$. But this implies that $A$ is either compressible or boundary parallel.

To see that $\gamma$ is annulus-busting in $M$, note that by (1) it is disk-busting. Now let $A$ be an annulus disjoint from $\gamma$ in $M$ and assume it is incompressible and not boundary parallel. By (2) it can be isotoped to be disjoint from the decomposing disk $D$. But this contradicts the annulus-busting assumption on each $\gamma_{i}$.

Lemma 5.9. For a genus $g>0$ handlebody, $H_{g}$, there is a non-separating simple closed curve $\eta_{g}$ which is 3-disk-busting and annulus-busting.

Proof. We prove the Lemma by induction on $g$.

Let $H_{1}$ be a solid torus and $\eta_{1}$ be a simple closed curve in $\partial H_{1}$ with winding number 3 in $H_{1}$. Then $\eta_{1}$ is clearly 3-disk-busting and annulus-busting in $H_{1}$ (an incompressible annulus in a solid torus is boundary parallel). This proves the Lemma when $g=1$. Let $b_{1} \subset \partial H_{1}$ be a band running between opposite sides of $\eta_{1}$. 
Let $\eta$ be two copies of $\eta_{1}$ in $H_{1}$. Then $\eta$ is also 3 -disk-busting and annulus busting in $H_{1}$. Let $b$ be a band connecting the two components of $\eta$. Let $\left(H_{2}, \eta_{2}\right)$ be the boundary plumbing of $\left(H_{1}, \eta_{1}\right)$ and $\left(H_{1}, \eta\right)$ gotten by identifying $b_{1}$ and $b$. By Lemma 5.8, $\left(H_{2}, \eta_{2}\right)$ is 3-disk-busting and annulus-busting. $H_{2}$ is a genus 2 handlebody and $\eta_{2}$ is connected and non-separating ( $b_{1}$ can be thought of as gotten from a curve $c_{1}$ intersecting $\eta_{1}$ once in $\partial H_{1}$, this curve perturbed slightly shows $\eta_{2}$ to be non-separating). Thus we have verified the Lemma when $g=2$.

Assume $\eta_{g}$ in $H_{g}$ satisfies the Lemma, for some $g>1$. Let $c_{g}$ be a simple closed curve in $\partial H_{g}$ intersecting $\eta_{g}$ once. A neighborhood of $c_{g}$ broken at $\eta_{g}$ becomes an embedded band $b_{g}$ that runs from one side of $\eta_{g}$ to the other. Let $\left(H_{g+1}, \eta_{g+1}\right)$ be the boundary plumbing of $\left(H_{1}, \eta\right)$ to $\left(H_{g}, \eta\right)$ along the bands $b$ and $b_{g}$. By Lemma 5.8. $\left(H_{g+1}, \eta_{g+1}\right)$ is 3-disk-busting and annulus-busting. $H_{g+1}$ is a genus $g+1$ handlebody and $\eta_{g+1}$ is connected and non-separating (as above, use a perturbed $c_{g}$ to verify that $\eta_{g+1}$ is non-separating). This verifies the Lemma for $g+1$ and the induction.

\subsection{Another 3-disk-busting and annulus-busting curve.}

Figure 6(left) shows a curve $\gamma_{2}$ in the boundary of a genus 2 handlebody $H_{2}$ that is disjoint from the curves $a(2, *, 0), b(2, *, 0)$ in each of Figure 7 (right) and (left). We show in Lemma 5.12 that this curve $\gamma_{2}$ is both 3disk-busting and annulus-busting. In section 5.4 for $g \geq 3$ we will boundary plumb $\left(H_{2}, \gamma_{2}\right)$ to $\left(H_{g-2}, \eta_{g-2}\right)$ along the bands $b_{\gamma}$ (also shown in Figure 7) and $b_{g-2}$ as in the construction of Lemma 5.9 to produce a new non-separating 3-disk-busting and annulus-busting curve $\gamma_{g}$ in the genus $g$ handlebody $H_{g}$ that is disjoint from curves $a(g, *, 0), b(g, *, 0)$ induced from $a(2, *, 0), b(2, *, 0)$.
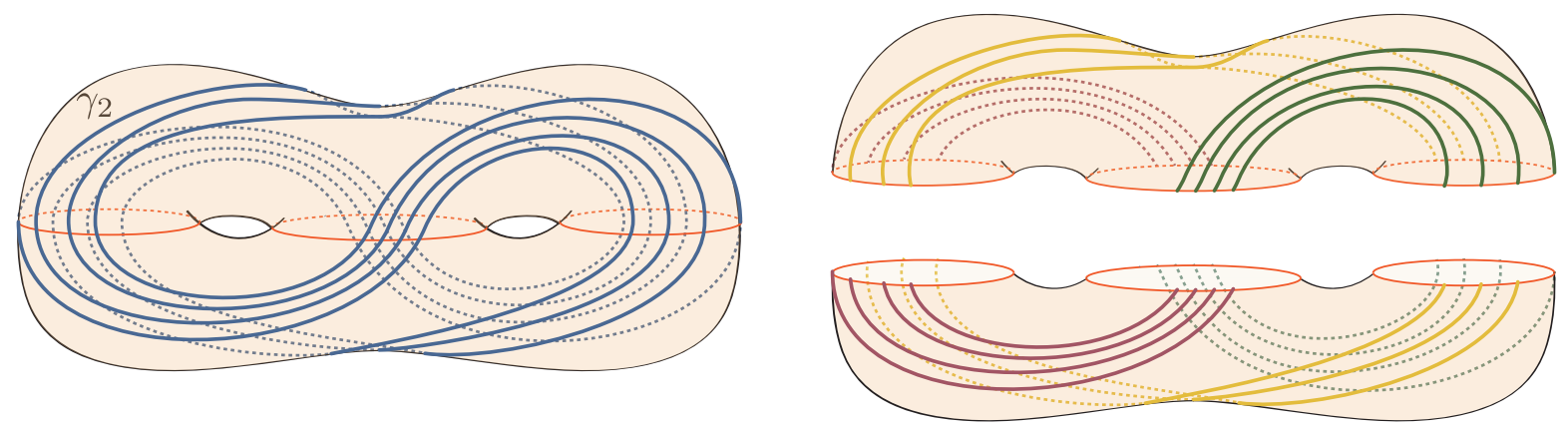

Figure 6: The curve $\gamma_{2}$ in the boundary of the genus 2 handlebody is shown with a compatible pants decomposition $\mathcal{P}$ (left). The handlebody is divided along $\mathcal{P}$, and the arcs of intersection of $\gamma_{2}$ with each pair of pants is colored according to isotopy classes.
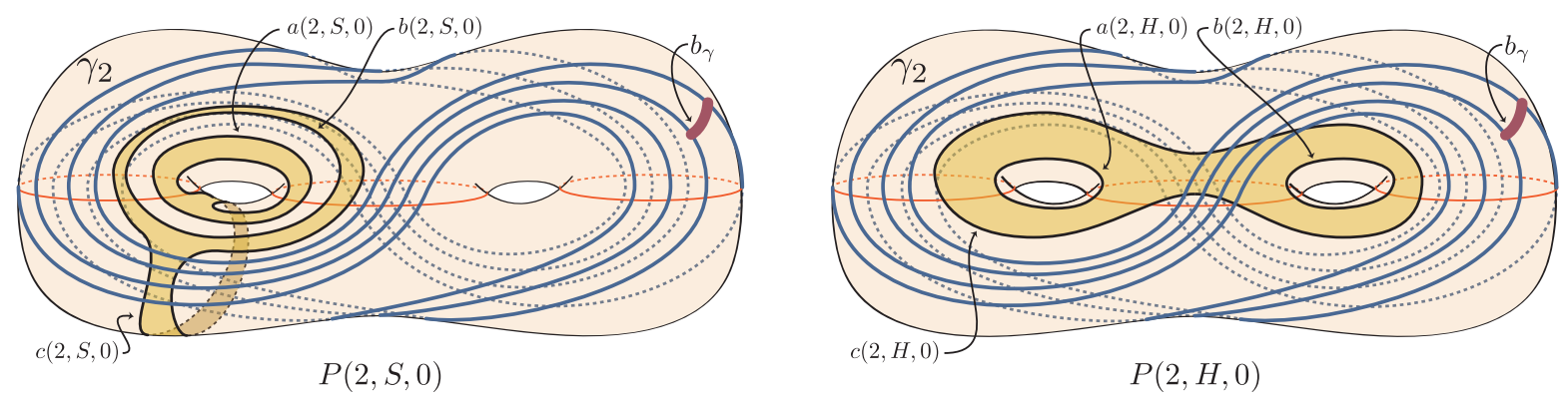

Figure 7: Seifert type and handlebody type pairs of pants in the boundary of $H_{2}$ along with the curve $\gamma_{2}$. A non-trivial band $b_{\gamma}$ connecting $\gamma_{2}$ from just one side is also shown. 
Lemma 5.10. Let $\gamma$ be a simple closed curve in the boundary of a genus 2 handlebody $H$. If $\gamma$ is disk-busting but not annulus-busting then $H[\gamma]$ contains an essential annulus.

Proof. Assume $A$ is a properly embedded essential annulus in $H$ that is disjoint from $\gamma$ and becomes inessential in $H[\gamma]$. Because $\gamma$ is disk-busting, the Handle Addition Lemma [CGLS87, Lemma 2.1.1], shows that $H[\gamma]$ is irreducible and boundary-irreducible.

First we show that $A$ must be separating in $H$. If $A$ were compressible in $H[\gamma]$, each component of $\partial A$ would have to be trivial in $\partial H[\gamma]$. This would then imply that $A$ is separating in $H$, since the boundary components of a non-separating annulus in $H$ are individually and jointly non-separating. On the other hand, if $A$ is boundary-parallel in $H[\gamma], A$ must be separating in $H$. Thus $A$ is separating.

A $\partial$-compression of $A$ in $H$ shows that $A$ may be viewed as the result of banding an essential separating disk to itself on one side. Thus $A$ divides $H$ into a solid torus and another genus 2 handlebody $H^{\prime}$. Since $A$ is not boundary-parallel in $H$, it must wind around this solid torus more than once. So if $\gamma$ were on the solid torus side of $A$, then $H[\gamma]$ would contain a lens space summand; but this cannot occur since $H[\gamma]$ is irreducible. Thus $\gamma$ must lie in $\partial H^{\prime}$. Then irreducibility similarly shows that $A$ cannot be compressible in $H[\gamma]$. So $A$ must be boundary-parallel in $H[\gamma]$. That is, $H^{\prime}[\gamma]$ must become a solid torus in which $A$ winds once. Hence $H[\gamma]$ is also a solid torus. But $H[\gamma]$ is boundary-irreducible.

Lemma 5.11. Let $\gamma$ be an essential simple closed curve in the boundary of a genus 2 handlebody $H$. If (the interior of) $H[\gamma]$ is a hyperbolic 3-manifold, then $\gamma$ is disk-busting and annulus-busting.

Proof. Assume the interior of $H[\gamma]$ is hyperbolic. Then it contains no properly embedded essential disks or annuli. If $\gamma$ were not disk-busting, then there would be a non-separating disk in $H$ disjoint from $\gamma$ that would become a non-separating disk in $H[\gamma]$. Thus $\gamma$ is disk-busting. Lemma 5.10 then implies that $\gamma$ must also be annulus-busting.

Lemma 5.12. The curve $\gamma_{2}$ in the boundary of the genus 2 handlebody $H$ as shown in Figure 6(left) is 3-disk-busting and annulus-busting.

Proof. Figure 6 (right) demonstrates that $\gamma_{2}$ is 3-seamed with respect to a compatible pair of pants decomposition of $H$. By Lemma 5.6, this means $\gamma_{2}$ is 3 -disk-busting. Figure 8 shows that $H\left[\gamma_{2}\right]$ is the exterior of the $5_{2}$ knot. Since this manifold is hyperbolic, Lemma 5.11 implies that $\gamma_{2}$ is annulus-busting.
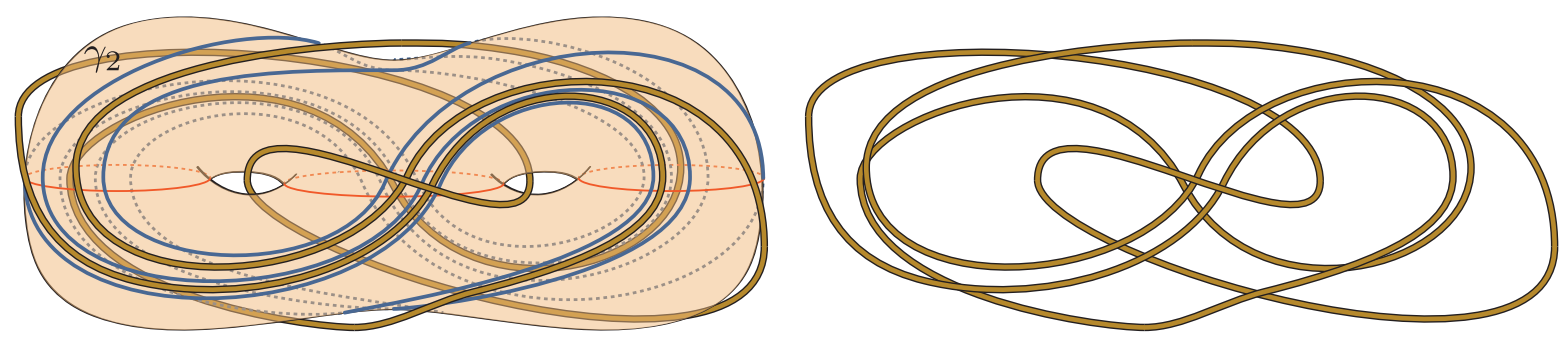

Figure 8: Attaching a 2-handle to the genus 2 handlebody $H_{2}$ along the curve $\gamma_{2}$ produces the exterior of the $5_{2}$ knot, a hyperbolic manifold. Shown (with and without $H_{2}$ ) is a knot disjoint from the standardly embedded $H_{2}$ and from a 2 -handle attached to $\gamma_{2}$. One easily confirms this is the $5_{2}$ knot.

\subsection{Disk and annulus hitting numbers}

Here we quantify the extent to which a knot is disk-busting or annulus-busting. 
Definition 5.13. (Disk Hitting Number) Let $M$ be a compact, connected, orientable manifold with boundary. Let $\mathcal{D}$ be the disk set of $M$, i.e. the set of properly embedded disks in $M$ that are not boundary-parallel. Assuming $\mathcal{D} \neq \emptyset$, the disk hitting number of $K$ in $M, \hbar_{D}(K)$, is the smallest number of times $K$ transversely intersects an essential disk in $M$. That is,

$$
\hbar_{D}(K)=\min _{D \in \mathcal{D}}|K \cap D|
$$

The disk hitting number is analogous to the wrapping number of a link in a solid torus. In a similar way, one can also define a hitting number for a knot in a 3-manifold relative to other homeomorphism types of surfaces. For our purposes, we only need the annular case.

Definition 5.14. (Annulus Hitting Number) Let $M$ be a compact, connected, orientable manifold with boundary. Let $\mathcal{A}$ be the annulus set of $M$, i.e. the set of incompressible, properly embedded annuli in $M$ that are not boundary-parallel. Assuming $\mathcal{A} \neq \emptyset$, the annular hitting number of $K$ in $M, \hbar_{A}(K)$, is the smallest number of times that $K$ transversely intersects an essential annulus in $M$. That is,

$$
\hbar_{A}(K)=\min _{A \in \mathcal{A}}|K \cap A| .
$$

\subsection{Handlebody, knot pairs with large hitting numbers}

The goal of this section is Proposition 5.18 which gives infinitely many knots in a genus $g$ handlebody admitting non-trivial handlebody or Seifert-type Dehn surgeries, distinguished by their hitting numbers. Proposition 5.19 shows that generically the exteriors of these knots are irreducible, $\partial$-irreducible, atoroidal, and anannular.

Figure 7 depicts two pairs of a genus 2 handlebody $H_{2}$ with a pair of pants in the boundary of $H_{2}$; on the left the pair $\left(H_{2}, P(2, S, 0)\right)$ is of Seifert type and on right the pair $\left(H_{2}, P(2, H, 0)\right)$ is of handlebody type (see Definitions 3.4 and 3.5. Also shown in each is a curve $\gamma_{2} \subset \partial H_{2}$ that is 3 -disk-busting and annulus-busting according to Lemma 5.12 .

Definition $5.15\left(\right.$ Of $\gamma_{g}, P(g, *, i)$, and $\left.M(g, *, i)\right)$. Let $b_{\gamma}$ be the band connecting $\gamma_{2}$ to the same side of itself in $\partial H_{2}$ as shown in Figure 7. Then, for $g \geq 3$, let $\gamma_{g}$ be the curve in the boundary of the genus $g$ handlebody $H_{g}$ obtained by boundary plumbing $\left(H_{g-2}, \eta_{g-2}\right)$ and $\left(H_{2}, \gamma_{2}\right)$ together along the bands $b_{g-2}$ and $b_{\gamma}$. (The triple $\left(H_{g-2}, \eta_{g-2}, b_{g-2}\right)$ are constructed in Lemma 5.9) By Lemma $5.8 \gamma_{g}$ is 3-disk-busting and annulus-busting.

Since $b_{\gamma}$ is disjoint from $P(2, *, 0)$ for $* \in\{S, H\}$, this boundary plumbing induces the pair of pants $P(g, *, 0)$ in the boundary of $H_{g}$. For each integer $i$, let $P(g, *, i)$ be the pair of pants obtained from $P(g, *, 0)$ by $i$ positive Dehn twists along $\gamma_{g} . P(g, *, i)$ has two boundary components which are disjoint from $\gamma_{g}$, label these $a(g, *, i), b(g, *, i)$. Label the third component $c(g, *, i)$. Then $a(g, S, i), b(g, S, i)$ are parallel and primitive in $H_{g} ; a(g, H, i), b(g, H, i)$ are jointly primitive in $H_{g}$. Furthermore, $c(g, *, i)$ is gotten from $c(g, *, 0)$ by Dehn-twisting along $\gamma_{g}$ in $\partial H_{g}$.

Recall from Section 2 the identifications of the genus 2 handlebody $H$ as a product $T \times[-1,1]$ for a oncepunctured torus $T$ and also as a product $P \times[-1,1]$ for a pair of pants $P$. The boundary components of $P$ are labelled $\partial_{-} P, \partial_{+} P, \partial_{0} P$. Recall that $K(\tau)$ is a knot in $T \times\{0\} \subset H=T \times[-1,1]$ obtained from an essential simple closed curve $\tau \subset T$. Fix a homeomorphism $\phi(*): P \rightarrow P(2, *, 0)$ that identifies $\partial_{-} P, \partial_{+} P$ with $a(2, *, 0), b(2, *, 0)$ in $P(2, *, 0)$. Let $\phi(g, *, i): P \rightarrow P(g, *, i)$ be the homeomorphism gotten by $\phi(*)$ followed by twisting along $\gamma_{g}$. To $\left(H_{g}, P(g, *, i)\right)$ constructed above, attach $H$ using $\phi(g, *, i)$ to identify $P \times\{-1\}$ with $P(g, *, i)$. Call the resulting genus $g$ handlebody $M(g, *, i)$. Let $K(\tau, g, *, i)$ be the knot in $M(g, *, i)$ that is the image of $K(\tau) \subset H$ under inclusion. 
We will apply Theorem 4.2 to show that for fixed $\tau(\neq \mu, \lambda)$ and $g \geq 2$

1. the disk hitting numbers of the knots $K(\tau, g, *, i) \subseteq M(g, *, i)$ go to infinity with $i$, and

2. the annular hitting numbers of the knots $K(\tau, g, *, i) \subseteq M(g, *, i)$ go to infinity with $i$.

To do this, let $\Sigma \times[0,1]$ be a collar neighborhood of $\partial M(g, *, 0)$, so that $\Sigma \times\{0\}=\partial M(g, *, 0)$. Let $J_{g}$ be the curve $\gamma_{g} \times\{1\} \subseteq \Sigma \times[0,1]$. There is an annulus that runs from $J_{g}$ to $\partial M(g, *, 0), \widehat{U}_{g}=\gamma_{g} \times[0,1]$. We identify $H \cong P \times I$ in $\Sigma \times[0,1]$ as $P(g, *, 0) \times[0,1 / 2]$. Then the knots $K(\tau, g, *, i) \subseteq M(g, *, i)$ are gotten from the knots $K(\tau, g, *, 0) \subseteq M(g, *, 0)$ by twisting $i$ times along the annulus $\widehat{U}_{g}$ (under the identification $\left.M(g, *, i) \cong H_{g} \cong M(g, *, 0)\right)$. This twisting is basically the same as the construction described in BGL13] and discussed in Definition 2.6 except that one boundary component of the annulus $\widehat{U}_{g}$ is in the boundary of the 3-manifold $M(g, *, 0)$. Specifically,

Definition 5.16. Let $N$ be a submanifold of $M(g, *, 0)$ that is disjoint from $J_{g}$. Let $\widehat{U}_{g} \times[0,1]$ be a product neighborhood of $\widehat{U}_{g}$ in $M(g, *, 0)$. Let $h_{i}: \widehat{U}_{g} \times[0,1] \rightarrow \widehat{U}_{g} \times[0,1]$ be a homeomorphism gotten by $i$ complete twists along $\widehat{U}_{g}$, where $h_{i}$ is the identity on $\widehat{U} \times\{0,1\}$. Define the submanifold $N$ twisted $i$ times along $\widehat{U}_{g}$ as $\left[N-\left(\widehat{U}_{g} \times[0,1]\right)\right] \cup h_{i}\left(N \cap\left(\widehat{U}_{g} \times[0,1]\right)\right)$. On the boundary of $M(g, *, 0), h_{i}$ induces $i$ Dehn twists along $\gamma_{g}$. The sign of the twist along $\widehat{U}, h_{i}$, is taken so that the induced twist on the boundary is a positive Dehn twist along $\tau_{g}$.

Above, we are twisting $N=K(\tau, g, *, 0)$ along $\widehat{U}_{g}$ to get $K(\tau, g, *, i)$.

Our application of Theorem 4.2 in Proposition 5.18 requires the following constraint on Möbius bands and essential annuli in the exterior of $J_{g}$.

Lemma 5.17. Let $(M, K)=(M(g, *, 0), K(\tau, g, *, 0))$ and $J_{g}$ be as above. Then $M_{J_{g}}$ is irreducible and boundary-irreducible. Assume that $\tau$ is not parallel to $\mu$ or $\lambda$ in $T$. Let $T_{J}$ be the torus component of $\partial M_{J_{q}}$. There is no incompressible annulus in $M_{J_{g}}$ disjoint from $K$ with one boundary component on $T_{J}$ and the other on $\partial M$. Furthermore, there is no Möbius band properly embedded in $M_{J_{g}}$ with boundary on $T_{J}$.

Proof. Assume for contradiction that $U^{\prime}$ is such an annulus, and let $U_{g}$ be the restriction of $\widehat{U}_{g}$ to $M_{J_{g}}$. Because $\gamma_{g}$ is disk-busting, $M_{J_{g}}$ is irreducible and boundary-irreducible. This implies that $U^{\prime}$ and $U_{g}$ can be isotoped in $M_{J_{g} \cup K}$ to have disjoint boundaries on $T_{J}$ and, in fact, isotoped in $M_{J_{g}}$ to be disjoint. Let $A$ be the properly embedded annulus in $M$ gotten by taking the union of $\widehat{U}_{g}$ and (an extension of) $U^{\prime}$ along $J_{g}$. A must be parallel into $\partial M$, otherwise there is an essential disk in $M$ disjoint from $A$ - contradicting that $\gamma_{g}$ is disk-busting. But this implies that $U_{g}$ and $U^{\prime}$ are properly isotopic in $M_{J_{g}}$. Thus, by an isotopy in a neighborhood of $\partial M$, we may take $\partial U^{\prime}=\gamma_{g}$. In particular, $\partial U^{\prime}$ is disjoint from $a(g, *, 0) \cup b(g, *, 0)$ and no arcs of $\partial U^{\prime}-c(g, *, 0)$ are parallel into $c(g, *, 0)$. Let $P^{\prime}=(P(g, *, 0) \times\{1 / 2\}) \cup(\partial P(g, *, 0) \times[0,1 / 2])$. Then $P^{\prime}$ is incompressible in $M_{J_{g}}$ (since $P^{\prime}$ is isotopic into $\partial M_{J_{g}}$ and $M_{J_{g}}$ is boundary-irreducible). So we may assume that $U^{\prime} \cap P^{\prime}$ contains no simple closed curves which are trivial in either $U^{\prime}$ or $P^{\prime}$. The $\operatorname{arcs}$ of intersection of $U^{\prime}$ with $P^{\prime}$ identify (in $U^{\prime}$ ) a $\partial$-compressing disk $D$ for $P^{\prime}$ in $M$ that intersects $\partial M$ in a component of $\gamma_{g}-c(g, *, 0)$. One checks that $D$ must then lie in $H$ and $D \cap \partial M$ is an essential arc in $P(g, *, 0)$ running from $c(g, *, 0)$ to itself. As $D$ comes from $U^{\prime}$, it is disjoint from $K$. As $\nu$ is isotopic to $c(g, *, 0)$ in $H, \tau$ then cannot be isotopic to $\nu$ in $T$. Lemma 2.11 implies that $\tau$ must be isotopic to $\mu$ or $\lambda$ in $T$ - contradicting our assumptions.

Now assume there were a Möbius band, $B$, in $M_{J_{g}}$. Note that $B$ must be $\partial$-incompressible in $M_{J_{g}}$ since $T_{J}$ does not bound a disk in $M_{J_{g}}$. Then $U_{g}$ can be isotoped in $M_{J_{g}}$ such that its boundary is disjoint from that of $B$. In fact, we may take $B$ to be disjoint from $U_{g}$ (they must intersect in simple closed curves parallel to the boundary in $B$ ). But then we can amalgamate $\widehat{U}_{g}$ with $B$ along $J_{g}$ to obtain a Möbius band, $\widehat{B}$, properly embedded in $M$. There must be a meridian disk in $M$ disjoint from $\widehat{B}$ and in particular from $\partial \widehat{U}_{g}$ in $M$. But this contradicts that $\gamma_{g}$ is disk-busting. 
The main result of this section is:

Proposition 5.18. Assume $g \geq 2$ and $\tau$ is not isotopic to $\mu$ or $\lambda$ in $T$. Consider the knot $K(\tau, g, *, i)$ in the handlebody $M(g, *, i)$ where $*=H, S$.

1. $K(\tau, g, H, i)$ admits a longitudinal surgery that is a handlebody.

2. $K(\tau, g, S, i)$ admits a longitudinal surgery which is a $D(p, q)$-Seifert space in union with $g-1$ onehandles when $\tau=\tau(p, q)$.

3. There is a function $N(\tau)$, i.e. independent of $g, *, i$, such that $b_{g}(K(\tau, g, *, i))<N(\tau)$.

4. There is a constant $N_{D}(\tau, *)>0$ such that $\max \left(\hbar_{D}(K(\tau, g, *, i)), 1\right) \geq|i| \cdot N_{D}(\tau, *)-1$, and hence $\hbar_{D}(K(\tau, g, *, i)) \rightarrow \infty$ as $i \rightarrow \infty$.

5. There is a constant $N_{A}(\tau, *)>0$ such that $\max \left(\hbar_{A}(K(\tau, g, *, i)), 1\right) \geq|i| \cdot N_{A}(\tau, *)-2$, and

Proof. Statements (1) and (2) follow directly from Proposition 3.8.

Fix $\tau, g$ and let $(M, K)=(M(g, *, 0), K(\tau, g, *, 0))$. Let $L=J_{g}=\gamma_{g} \times\{1\} \subseteq \Sigma \times[0,1], \widehat{U}=\widehat{U}_{g}$ be as above (preceding Lemma 5.17).

Statement (3). Put $\tau \subset H=P \times I$ in bridge position with respect to the height function of this product structure. Let $N(\tau)$ be the bridge number of this presentation. When included into $M, H$ can be regarded as residing within a collar neighborhood of $\partial M$ so that this product structure of $H$ is compatible with the product structure of the collar. Hence this puts $K$ in bridge position entirely within a collar neighborhood of $M$ that is disjoint from $L$, so the bridge number of $K$ in $M$ is at most $N(\tau)$. As the $\operatorname{knots}\{K(\tau, g, *, i), i \in \mathbb{Z}\}$ are obtained from $K$ by twisting along the vertical annulus $\widehat{U}$, which preserves the height in the collar, the bridge numbers of these knots is also at most $N(\tau)$. This is statement (3) of the Proposition, noting that the bound is independent of $g, *, i$.

For statements (4) and (5) we need a catching surface in order to apply Theorem 4.2 . Let $\bar{Q}_{2}$ be the meridian disk of $\mathrm{H}_{2}$ whose boundary is the leftmost curve of the pants decomposition $\mathcal{P}$ in Figure 6 . Observe that $\gamma_{2}$ intersects $\bar{Q}_{2}$ algebraically once (with appropriate orientation) but geometrically seven times. Let $\bar{Q}_{g}$ be the corresponding disk gotten by the inclusion of $H_{2}$ into $H_{g}$. We also see algebraic intersection number one (with appropriate orientations) and geometric intersection number seven between $\gamma_{g}$ and $\bar{Q}_{g}$.

It follows that in $M, L$ and $\bar{Q}_{g}$ also algebraically intersect once but geometrically seven times. Keeping $L$ fixed, tube oppositely oriented intersections of $L$ and $\bar{Q}_{g}$ together to produce an oriented surface that $L$ intersects coherently and then isotop $K$ in the complement of $L$ to intersect this surface transversally. Let $Q$ be the restriction of the tubed $\bar{Q}_{g}$ to the exterior of $K \cup L$ in $M, X=M_{K \cup L}$. Note that $\chi(Q)=$ $\chi\left(\bar{Q}_{g}-N(K \cup L)\right) \leq \chi\left(\bar{Q}_{g}-N(L)\right)=-6$. Let $T_{K}$ be the component of $\partial X$ corresponding to $K$ and $T_{L}$ be that corresponding to $L$. Observe that, by construction, $Q$ intersects $T_{L}$ in a single component, a meridian of $L$. $Q$ will serve then as a catching surface for $(F, K, L)$ where $F$ is a properly embedded surface in $X$ whose boundary on $T_{L}$ is not meridional, see Definition 4.1. Note that being a catching surface does not require $Q$ to be incompressible or $\partial$-incompressible in $X$. The surface $F$ will differ in (4) and (5).

Statement (4). Recall from the discussion preceding Lemma 5.17, that we may think of the knots $K(\tau, g, *, i)$ as gotten from $K=K(\tau, g, *, 0)$ by twisting $i$ times along the annulus $\widehat{U}_{g}$ in $M$. Let $U_{g}$ be the restriction of this annulus to $X$. Let $\bar{F}_{i}$ be a meridian disk of $M(g, *, i)$ that, among all meridian disks of $M(g, *, i)$, intersects $K(\tau, g, *, i)$ minimally. This geometric intersection number is $\hbar_{D}(K(\tau, g, *, i))$. Furthermore, subject to this minimality assume that $\bar{F}_{i}$ intersects $L$ minimally, a non-empty intersection since $\gamma_{g}$ is disk-busting in $M$. Let $F_{i}$ be the restriction of $\bar{F}_{i}$ to the exterior, $X_{i}$, of $K(\tau, g, *, i) \cup L$ in $M(g, *, i)$. Then $F_{i}$ is incompressible in $X_{i}$. Note that twisting along the annulus $\widehat{U}_{g}$ induces a homeomorphism between $X$ and $X_{i}$. Under this homeomorphism we view $F_{i}$ as a surface properly embedded in $X$. We are now in the context of Section 4 with the surfaces $F=F_{i}$ and $Q$ properly embedded in $X$. In that notation 
$\alpha_{K}, \beta_{K}$ are meridians of $K$ and consequently $\Delta_{K}=0$ so that $\Delta_{K}^{\prime}=1$. As $\beta_{L}$ is a meridian of $L$ and $\alpha_{L}$ is obtained from a meridian of $L$ by Dehn-twisting longitudinally $-i$ times in $T_{L}$ along $\partial U_{g}$, we have $\Delta_{L}=|i|$. In particular, when $i \neq 0$ then $\alpha_{L}$ is not a meridian of $L$, so $Q$ is a catching surface for $(F, K, L)$. Here $f_{M}=f_{M}^{\prime}=1, \chi(\widehat{F})=2, \chi(Q)<0$ and $f_{K}=\hbar_{D}(K(\tau, g, *, i))$. (Recall that $\widehat{F}$ is the surface $F$ with all of its boundary components capped off with disks.) Finally, $F$ is incompressible in $X$, hence it is $\partial$-incompressible at $T_{L}$, and $\left|F \cap T_{L}\right| \neq 0$. Then Theorem 4.2 along with Lemma 5.17 implies that

$$
\max \left(\hbar_{D}(K(\tau, g, *, i), 1) \geq \frac{|i|}{36|\chi(Q)|}-1 .\right.
$$

When $i=0$, note that the above holds trivially. Observe that $\chi(Q)$ depends on both $\tau$ and $* \in\{H, S\}$, but is independent of $g$ and $i$. For statement (4) we then set $N_{D}(\tau, *)=\frac{1}{36|\chi(Q)|}>0$. Then, as $|i| \rightarrow \infty$, $\max \left(\hbar_{D}(K(\tau, g, *, i)), 1\right) \geq|i| N_{D}(\tau, *)-1$ implies $\hbar_{D}(K(\tau, g, *, i)) \rightarrow \infty$.

Statement (5). Repeat the argument above taking $\bar{F}_{i}$ to be an essential annulus. More specifically, let $\bar{F}_{i}$ be a properly embedded essential annulus in $M(g, *, i)$ that, among all essential annuli in $M(g, *, i)$ intersects $K(\tau, g, *, i)$ minimally. This intersection number is $\hbar_{A}(K(\tau, g, *, i))$. Furthermore, subject to this minimality assume that $\bar{F}_{i}$ intersects $L$ minimally, a non-empty intersection since $\gamma_{g}$ is annulus-busting. As above, let $F_{i}$ be the restriction of $\bar{F}_{i}$ to $X_{i}$ and, via the homeomorphism between $X$ and $X_{i}$, regard $F_{i}$ as an incompressible surface $F$ properly embedded in $X$. Again, we have $\Delta_{K}=0$ so that $\Delta_{K}^{\prime}=1, \Delta_{L}=|i|$, and $\chi(\widehat{F})=2$, but now $f_{M}=f_{M}^{\prime}=2$, and $f_{K}=\hbar_{A}(K(\tau, g, *, i))$. When $i \neq 0$, then $Q$ is a catching surface for $(F, K, L)$.

Then Theorem 4.2 along with Lemma 5.17 implies that

$$
\max \left(\hbar_{A}(K(\tau, g, *, i)), 1\right) \geq \frac{|i|}{72|\chi(Q)|}-2 .
$$

For statement (5) we then set $N_{A}(\tau, *)=\frac{1}{72|\chi(Q)|}$. Then, as $|i| \rightarrow \infty, \max \left(\hbar_{A}(K(\tau, g, *, i)), 1\right) \geq|i| N_{A}(\tau, *)-$ 2 implies $\hbar_{A}(K(\tau, g, *, i)) \rightarrow \infty$.

Section 3 talks about when a pair of pants in the boundary of a handlebody is of strong Seifert type or strong handlebody type, a notion which allows us to get better control over the exteriors of the knots constructed.

Proposition 5.19. For $g \geq 2$, there is a constant $N_{\text {strong }}$ such that when $|i|>N_{\text {strong }}$,

- $M(g, *, i)$ is of strong Seifert type or strong handlebody type, and

- when $\tau \notin\{\lambda, \mu, \nu, \lambda-\mu, \lambda+\nu, \mu+\nu\}$, the exterior of $K(\tau, g, *, i)$ in $M(g, *, i)$ is irreducible, atoroidal, $\partial$-irreducible, and anannular.

Proof. The pair $\left(H_{g}, c(g, *, i)\right)$ is homeomorphic to the pair $(M(g, *, i), K(\nu, g, *, i))$ since $K(\nu)$ is isotopic in $H$ to $\partial_{0} P=c(g, *, i)$. By Proposition 5.18(4), for any $n$ there is an $N_{D}(\nu, n)$ such that if $|i|>N_{D}(\nu, n)$, $\hbar_{D} K(\nu, g, *, i)>n$ in $M(g, *, i)$ (taking the larger of the constants over $\left.*=H, S\right)$. Thus for $|i|>N_{D}(\nu, n)$, $c(g, *, i)$ is $n$-disk-busting in $H_{g}$. Similarly, by Proposition 5.18 $(5)$, there is a constant $N_{A}(\nu, n)$ independent of $g$ such that for any $n \in \mathbb{N}$, if $|i|>N_{A}(\nu, n), \hbar_{A} K(\nu, g, *, i)>n$ in $M(g, *, i)$. Thus for $|i|>N_{A}(\nu, n)$, $c(g, *, i)$ is $n$-annulus-busting in $H_{g}$.

Set $N_{\text {strong }}=\max \left(N_{D}(\nu, 3), N_{A}(\nu, 1)\right)$ and assume $|i|>N_{\text {strong. }}$. Then $c(g, *, i)$ is 3 -disk-busting and annulus-busting. Lemma 3.6 then implies that $\left(H_{g}, P(g, *, i)\right)$, and hence $M(g, *, i)$, is of strong Seifert type or strong handelbody type. Proposition 3.12 completes the proof. 


\section{Bridge number bounds}

In this section we compute a lower bound on $b_{g}(K(\tau, g, *, i))$ in $M(g, *, i)$ for each $i$, Proposition 6.7 We then combine this with Propositions 5.18 and 5.19 to find the infinite families of knots for our main result Theorem 6.8. Recall that in Section 2, $\tau(\kappa, \alpha, n)$ means that the curve $\tau$ in $T \times\{0\}(H=T \times[-1,1])$ is gotten from $\kappa$ by Dehn twisting $n$ times along $\alpha$. Thinking of $\tau$ as a knot, $K(\tau)$, in $H$, we alternatively think of $K(\tau)$ as gotten by twisting $n$ times the knot $K(\kappa)$ along the annulus $\widehat{R}(\alpha)$ in $H$, see Definition 2.6 Recall that $\widehat{R}(\alpha)$ is the product $\alpha \times[-1 / 2,1 / 2]$ in $T \times[-1,1]$, and $L_{-}, L_{+}$are the components of $\partial \widehat{R}(\alpha)$. When the curve $\alpha$ is clear, we will only write $\widehat{R}$ for $\widehat{R}(\alpha)$.

Lemma 6.1. When $\alpha$ is parallel to $\nu, L_{+}$and $L_{-}$are isotopic in $H$ to $\partial_{0} P$. The slope that $\widehat{R}(\alpha)$ determines on $\partial N\left(L_{+}\right)$and on $\partial N\left(L_{-}\right)$is distance one (Definition 2.9) from the slope describing the parallelism to $\partial_{0} P$.

Proof. The first statement is obvious from the definition of $L_{+}$and $L_{-}$. By direct inspection, the slope of $\partial R$ on $\partial N\left(L_{+}\right)$, say, is distance one from the surface slope of $\partial M$; refer to Figure 2.

Recall from Definition 5.15 that $M(g, *, i)=(H, P) \cup_{P}\left(H_{g}, P(g, *, i)\right)$. Below we will refer to $P$ as the properly embedded copy of $P$ separating $H$ from $H_{g}$. Under the inclusion of $H$ in $M(g, *, i)$, we consider $K(\tau), K(\kappa), \widehat{R}(\alpha)$ as living in $M(g, *, i)$. Following [BGL13, a genus $g$ splitting of the genus $g$ handlebody $M(g, *, i)$ is the decomposition of $M(g . *, i)$ into a union $H_{1} \cup_{\Sigma} H_{2}$ where $H_{1}$ is a genus $g$ handlebody, $\Sigma$ is a surface of genus $g$, and $H_{2} \cong \Sigma \times I$. This is the standard splitting of $M(g, *, i)$.

Lemma 6.2. Assume that $M=M(g, *, i)$ is of strong handlebody or strong Seifert type. If $\alpha$ is not parallel to $\mu$ or $\lambda$, then $\widehat{R}(\alpha)$ is not isotopic into the standard splitting of $M$.

Proof. That the type is strong guarantees that $P$ is incompressible in $M, \partial$-incompressible in $M-H$ (for the handlebody type see Lemma 3.6, and that $\partial_{0} P$ is disk-busting in $M$. Suppose first that $\alpha$ is not parallel to $\mu, \lambda$, or $\nu$. We will show that the core of $\widehat{R}$ is not isotopic into $\partial M$. Suppose that it is, so that there is an annulus $A$ with one boundary component, $\partial_{1} A$, on the core of $\widehat{R}$ and the other, $\partial_{2} A$, lying on $\partial M$. Choose $A$ to minimize $|A \cap P|$ and note that $A$ is incompressible in $M$ since $L_{+}$and $L_{-}$are nontrivial knots. Because $P$ is also incompressible, there are no trivial simple closed curves of intersection of $A$ with $P$. Among arcs of intersection of $A \cap P$, choose $a$ to be outermost in $A$ so that it cuts off a disk $D \subseteq A$ with int $D \cap P=\emptyset, \partial D=a \cup b$, and $b \subseteq \partial M$. If $b$ is trivial in $\partial M-P$ we can isotop $A$ to reduce $|A \cap P|$. By the incompressibility of $\partial M-P$, Lemma 3.6, $a$ is not trivial in $P$. Therefore $D$ must be a $\partial$-compressing disk for $P$ in $M$ that is disjoint from $K(\alpha)$, the core of $\widehat{R}$. As $P$ is $\partial$-incompressible in $M-H, D$ must lie in $H$. But by Lemma 2.11 and our restrictions on $\alpha$, no such disks exist. Therefore $A \cap P=\emptyset$, which implies that $L_{+}$and $L_{-}$are isotopic to a component of $\partial P$. Since $H \cong T \times I, \alpha$ is isotopic in $T$ to $\mu, \lambda$, or $\nu$.

Now suppose that $\alpha$ is parallel to $\nu$ and $\widehat{R}$ is isotopic into the standard splitting surface $\Sigma$ for $M$. After an isotopy of $\widehat{R}$ into $\Sigma$, observe that $\Sigma-L_{+}$is incompressible because $L_{+}$is disk-busting in $M$ (it is isotopic to $\nu$ and hence to $c$ ). Let $\gamma$ be the framing on $L_{+}$induced by $\Sigma$, which is the same as that from $\widehat{R}$. Since $M \backslash \Sigma$ is the product between $\Sigma$ and $\partial M$ and the handlebody bounded by $\Sigma, M_{L_{+}}(\gamma)$ can be viewed as the union of these two pieces each with a 2-handle attached along the imprint of $L_{+}$. By the Handle Addition Lemma CGLS87, Lemma 2.1.1], the resulting boundary $\Sigma^{\prime}$ of the handlebody with a 2-handle attached along $L_{+}$is incompressible into that side of $\Sigma^{\prime}$. The other side of $\Sigma^{\prime}$ is a compression body in which $\Sigma^{\prime}$ is also incompressible. Thus $\Sigma^{\prime}$ is an incompressible surface in $M_{L_{+}}(\gamma)$. Recall that when $\alpha$ is parallel to $\nu$, $L_{+}$is isotopic to $\partial_{0} P$. However, by Lemma 6.1, the framing, $\gamma$, of $\widehat{R}$ on $L_{+}$is distance one from the surface framing by $\partial M$. Therefore, surgery on $L_{+}$at the slope $\gamma$ yields a handlebody. Handlebodies contain no closed incompressible surfaces, and so $\widehat{R}$ is not isotopic into $\Sigma$. 
Let $\tau=\tau(\kappa, \alpha, n)$ and recall the handlebody/knot pair $(M(g, *, i), K(\tau, g, *, i))$ of section 5.4 Under the inclusion of $H$ in $M(g, *, i)$, we consider $K(\kappa), K(\alpha)$ as well as the annulus $\widehat{R}(\alpha)$ and its boundary $L_{-}, L_{+}$ as embedded in $M(g, *, i)$. Then the knot $K(\tau, g, *, i)$ is gotten from $K(\kappa, g, *, i)$ by twisting $n$ times along the annulus $\widehat{R}(\alpha)$. Write $\mathcal{L}$ for the link $K(\kappa) \cup L_{-} \cup L_{+}$in $M(g, *, i)$ and $M(g, *, i)_{\mathcal{L}}$ for its exterior. Denote by $T_{+}, T_{-}, T_{\kappa}$ the components of $\partial M(g, *, i)_{\mathcal{L}}$ corresponding to $L_{+}, L_{-}, K(\kappa)$. We want to find a catching surface, $Q$, as defined in [BGL13, Definition 2.1], for the pair $(\widehat{R}(\alpha), K(\kappa))$ in $M(g, *, i)$. We repeat that definition in this context.

Definition 6.3. Let $Q$ be an oriented surface properly embedded in $M(g, *, i)_{\mathcal{L}}$. We say that $Q$ catches the pair $(\widehat{R}(\alpha), K(\kappa))$ if

- $\partial Q \cap T_{i}$ is a non-empty collection of coherently oriented parallel curves on $T_{i}$ for each $i \in\{+,-\}$; and

- $\partial Q$ intersects both $T_{+}$and $T_{-}$in slopes different than $\partial R(\alpha)$.

Lemma 6.4. Let $\tau=\tau(\kappa, \alpha, n)$. The annulus/knot pair $(\widehat{R}(\alpha), K(\kappa))$ in $M(g, *, i)$ is caught by a surface $Q$ with $\chi(Q)<0$.

1. Assume $\alpha$ is not parallel to $\mu, \lambda$ or $\nu$ in $T$ and $g \geq 3$. Then $Q$ intersects $T_{+}, T_{-}$in meridians, and if $Q$ intersects $T_{\kappa}$ at all it does so in meridians.

2. When $\alpha$ is not parallel to $\mu, \lambda$ or $\nu$ and $g=2, Q$ can be taken so that $\chi(Q)$ depends on $\kappa, \alpha$ but not on $i$. Then $Q$ intersects $T_{+}, T_{-}$in meridians, and if $Q$ intersects $T_{\kappa}$ at all it does so in meridians.

3. When $\alpha=\nu$ and $g \geq 2, Q$ can be taken so that $\chi(Q)$ depends on $\kappa, \alpha$ but not on $i$. Furthermore, the intersection of $Q$ with $T_{-}$is in meridians, with $T_{\kappa}$ in meridians (if non-empty), and with $T_{+}$in a single curve which is distance one from both the meridional slope and the slope induced by $\widehat{R}$.

Proof. Recall from section 5.4 that there is an annulus $\widehat{U}_{g}$ in $M(g, *, 0)$, gotten from the trace of an isotopy of $\gamma_{g}$ into the interior of $M(g, *, 0)$, such that the pair $(M(g, *, i), K(\tau, g, *, i))$ is gotten from the pair $(M(g, *, 0), K(\tau, g, *, 0))$ by twisting along $\widehat{U}_{g}$. Similarly, the pair $(\widehat{R}(\alpha), K(\kappa))$ in $M(g, *, i)$ is obtained from the pair $(\widehat{R}(\alpha), K(\kappa))$ in $M(g, *, 0)$ by twisting along $\widehat{U}_{g}$.

We first assume that $\alpha$ is not parallel to $\nu$ (or by assumption to either $\mu$ or $\lambda$ ). Let $D$ be the disk in $M(g, *, i)$ whose boundary is the middle curve of Figure 6(left) where $D \subset H_{2} \subset H_{g} \cong M(g, *, i)$.

Claim 6.5. The algebraic intersection number of $D$ with each of $L_{+}$and $L_{-}$in $M(g, *, i)$ is non-zero.

Proof. In the language of section 2 we may take $D \cap H=D_{+} \cup D_{-} \cup t D_{s}$ where $D_{s}$ is a separating product disk in $H$ in $M(g, *, i)$ and $t D_{s}$ means $t$ parallel copies of $D_{s}$ for a non-negative integer $t$. We may take $t=32 i$ when $*=H$ and $t=64 i$ when $*=S$. (Note: for $g=2, P(2, *, 0)$ is pictured in Figure 7, and $P(2, *, i)$ is gotten by twisting along $\widehat{U}_{2}$ ). The algebraic intersection number of $L_{+}$or $L_{-}$with $D$ then depends only on its intersections with $D_{-} \cup D_{+}$with the appropriate orientation. This is $\pm \Delta(\alpha, \mu) \pm \Delta(\alpha, \lambda)$ by Lemma 2.10 - which is non-zero as long as $\alpha \neq \tau(1,1), \tau(1,-1)$. By inspection one sees that the algebraic intersection number of $D$ with $\tau(1,1)=\nu=c(g, *, i)$ is zero. The algebraic intersection number of $\tau(1,-1)$ with the oriented $D_{-} \cup D_{+}=D_{\mu} \cup D_{\lambda}$ is different than that of $\tau(1,1)$, hence non-zero. This gives the conclusion.

Tube oppositely oriented intersections of $D$ along $L_{+}\left(L_{-}\right)$so that all intersections with $L_{+}\left(L_{-}\right)$have the same sign. Let $Q$ be the intersection of this surface with the exterior, $M(g, *, i)_{\mathcal{L}}$, of $\mathcal{L}=K(\kappa) \cup L_{+} \cup L_{-}$ in $M(g, *, i)$. Orienting $Q$, we see that $\partial Q$ is a non-empty, coherently oriented collection of meridians on the components, $T_{+}, T_{-}$, of $\partial M(g, *, i)_{\mathcal{L}}$. Note that a catching surface does not need to be incompressible or $\partial$-incompressible. Thus $Q$ becomes a catching surface for the annulus/knot pair $(\widehat{R}, K(\kappa))$ in $M(g, *, i)$. Note that $\chi(Q)<0$ and depends on $\kappa, \alpha, i$. This is conclusion (1) of the Lemma. 
We now modify the surface $Q$ to make its Euler characteristic independent of $i$ when $g=2$. First we consider $(\widehat{R}(\alpha), K(\kappa))$ in $M(2, *, 0)$. As above, we assume that $\alpha \neq \mu, \lambda, \nu . P(2, *, 0)$ is given in Figure 7 . Let $D$ be the disk whose boundary is the middle curve of Figure 6(left), and attach four bands to $D$ to obtain $\bar{Q}$, properly embedded in $M(2, *, 0)$, whose boundary does not meet the disk-busting curve $\gamma_{2}$. See Figure 9 .

Claim 6.6. The algebraic intersection number of $\bar{Q}$ with each of $L_{+}$and $L_{-}$in $M(2, *, 0)$ is non-zero.

Proof. In the language of section $2, \bar{Q} \cap H=D_{+} \cup D_{-} \cup t D_{s}$ where $D_{s}$ is a separating disk in $H$ and where we may take $t=4$ when $*=H$ and $t=8$ when $*=S$. The algebraic intersection number of $L_{+}$or $L_{-}$with $\bar{Q}$ is then $\pm \Delta(\alpha, \mu) \pm \Delta(\alpha, \lambda)$ by Lemma 2.10 - which is non-zero as long as $\alpha \neq \tau(1,1), \tau(1,-1)$. As in the proof of Claim 6.5. one sees that the algebraic intersection number of $\bar{Q}$ with $\tau(1,1)=\nu$ is zero, hence with $\tau(1,-1)$ is non-zero.

Tube oppositely oriented intersections of $\bar{Q}$ along $L_{+}\left(L_{-}\right)$, within $H$ of $M(2, *, 0)$, so that all intersections with $L_{+}\left(L_{-}\right)$have the same sign. Let $Q$ be the intersection of this tubed $\bar{Q}$ with the exterior, $M(2, *, 0)_{\mathcal{L}}$, of $\mathcal{L}=K(\kappa) \cup L_{+} \cup L_{-}$in $M(2, *, 0)$. Orienting $Q$, we see that $\partial Q$ is a non-empty, coherently oriented collection of meridians on the components, $T_{+}, T_{-}$, of $\partial M(2, *, 0)_{\mathcal{L}}$, and any intersections with $T_{\kappa}$ will be in meridians. Thus $Q$ becomes a catching surface for the annulus/knot pair $(\widehat{R}, K(\kappa))$ in $M(2, *, 0)$. Note that $\chi(\bar{Q})<0$. As $\bar{Q}$ is disjoint from $\gamma_{2}$, we may take $J_{2}$ disjoint from $Q$. After twisting along $\widehat{U}_{2}$, we see the image of $Q$, which we also refer to as $Q$, as a catching surface for $(\widehat{R}, K(\kappa))$ in $M(2, *, i)$. The boundary of $Q$ on each of $T_{-}, T_{+}, T_{\kappa}$ is still a collection of meridians, and $\chi(Q)$ is independent of $i$ as desired when $g=2$. This is conclusion (2) of the Lemma.
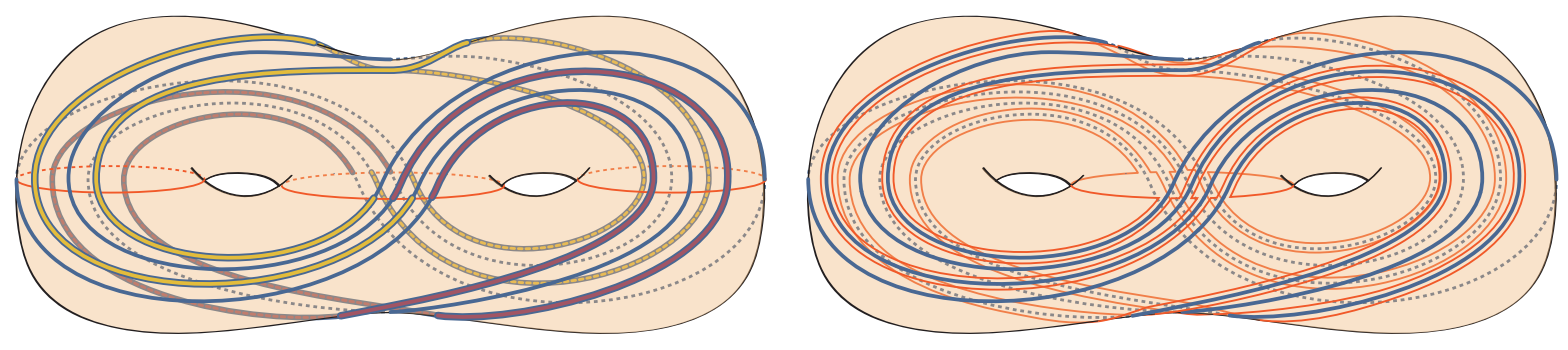

Figure 9: (Left) Four subarcs of $\gamma_{2}$ that connect the central meridian disk to itself from the same side are highlighted. (Right) The boundary of the resulting orientable surface $\bar{Q}$ is shown to be disjoint from $\gamma_{2}$.

We are left to consider the case that $\alpha$ is parallel to $\nu$ in $T$ in any genus $g$. We will first exhibit a 3-punctured sphere $\bar{Q}$ in the little handlebody $H=P \times I$ with boundary $a \times\{1\} \cup b \times\{1\} \cup L_{+}$that is intersected once by $L_{-}$and some number of times by $K(\kappa)$. Since $L_{+}$is isotopic to $\nu$ which is isotopic to $c \times\{1\}$, we may take $\bar{Q}$ to be the result of isotopy of $P \times\{1\}$ that fixes $a \times\{1\} \cup b \times\{1\}$ and pulls the rest into the interior of $P \times I$. See Figure 10. Because the framing that $T \times\{0\}$ gives $\nu$ differs from the framing that $P \times\{1\}$ gives $c$ by 1 , we see that $L_{-}$intersects $\bar{Q}$ just once. Then we take $Q$ to be the surface $\bar{Q}-N\left(L_{+} \cup L_{-} \cup K(\kappa)\right)$. Now we may attach $P \times I$ (along $P \times\{-1\})$ to $P(g, *, i)$ so that $Q$ becomes a catching surface for $(\widehat{R}(\alpha), K(\kappa)$ ) in $M(g, *, i)$. Then $\chi(Q)$ is independent of $i$. Furthermore, $Q$ intersects $T_{-}$in a single meridian, intersects $T_{\kappa}$ in meridians (if at all), and intersects $T_{+}$in a single component whose distance from the meridian and from $\partial \widehat{R}$ is one. This is conclusion (3).

We can now give lower bounds on the bridge numbers of the knots $K(\tau, g, *, i)$. These will be independent of $i$ when $g=2$ or $\alpha=\nu$.

Proposition 6.7. Fix a genus $g$, and let $N_{\text {strong }}$ be as in Proposition 5.19, Let $\kappa, \alpha$ be curves in $T$ such that $\alpha$ is not parallel to $\mu$ or $\lambda$ in $T$. Let $\tau=\tau(\kappa, \alpha, n)$. For $|i|>N_{\text {strong, }}$, there is a positive constant 

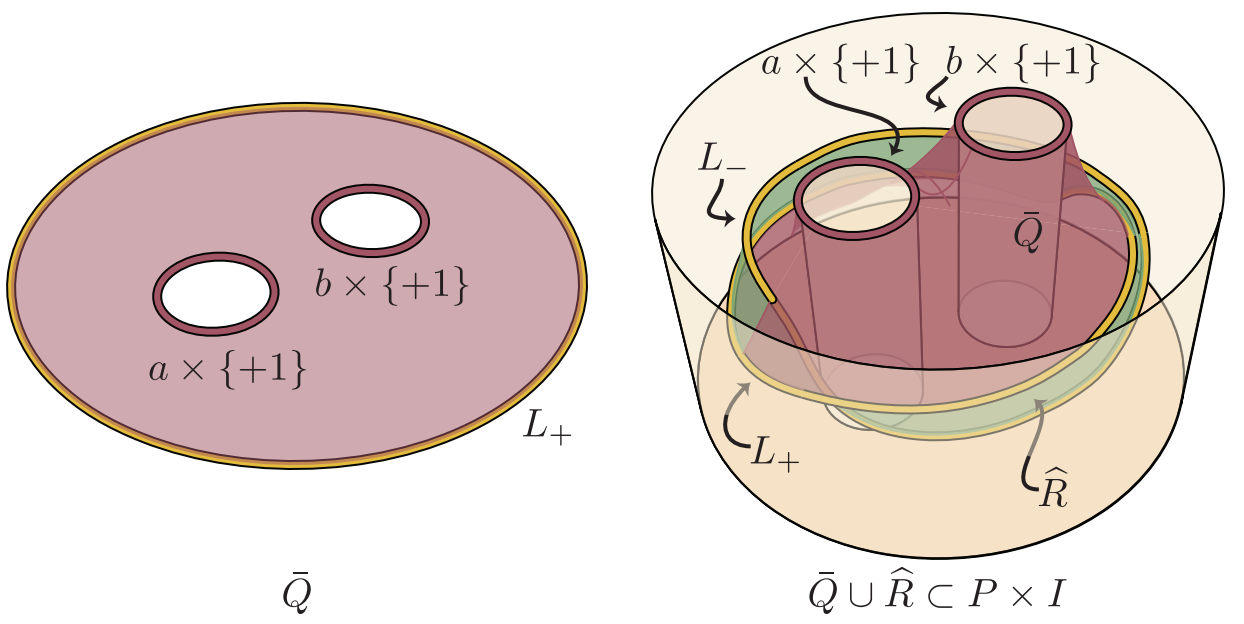

Figure 10: The 3-punctured sphere $\bar{Q}$ resides in $P \times I$ with $\widehat{R}$ as shown. Compare with $\nu \subset T \times\{0\}$ in Figure 2

$C=C(g, \alpha, \kappa, i)$ such that

$$
b_{g}(K(\tau, g, *, i)) \geq \frac{1}{2}(|n| C-2 g) .
$$

When either $g=2$ or $\alpha=\nu$, the constant $C$ can be chosen to be independent of $i$ (given $|i|>N_{\text {strong }}$ ).

Proof. This follows by applying [BGL13, Theorem 1.3] to the annulus/knot pair $(\widehat{R}(\alpha), \kappa)$ in $M=M(g, *, i)$. Assume $|i|>N_{\text {strong. }}$. By Proposition 5.19 and Lemma $6.2 \widehat{R}(\alpha)$ is not isotopic into the genus $g$ splitting of $M$. Furthermore, by Lemma 3.11 there is no essential annulus in $M_{\mathcal{L}}$ with one boundary component on each of $T_{+}$and $T_{-}$(recall the standing assumption, see Definition 2.6. that $\kappa$ and $\alpha$ are not isotopic in $T$.). Let $Q$ be the catching surface of Lemma 6.4 for the annulus/knot pair $(\widehat{R}(\alpha), \kappa)$ in $M$. Since the components of $Q$ are meridional on $T_{\kappa}$, and either meridional or longitudinal and distance one from the slope of the framing induced by $\widehat{R}(\alpha)$ on $T_{+}$and $T_{-}$, then, in the notation of Theorem 1.3 of [BGL13], $\Delta_{K}$ is zero, $\left|p_{1}\right|,\left|p_{2}\right|=1$, and $\left|q_{1}\right|,\left|q_{2}\right| \leq 1$. As $\chi(Q)$ is negative we conclude that

$$
\left.b_{g}(K(\tau, g, *, i))\right) \geq \frac{1}{2}\left(\frac{|n|}{-36 \chi(Q)}-2 g\right) .
$$

Noting that $\chi(Q)$ depends only on $\alpha, \kappa, g, i$, and that, when $g=2$ or $\alpha=\nu, \chi(Q)$ depends only on $\alpha, \kappa, g$, this becomes the statement of the Proposition.

Let the curves $\kappa$ and $\alpha$ be $(r, s)$ and $(t, v)$ curves with respect to the basis on $T$ given by $\mu$ and $\lambda$. The result of $n$ Dehn twists of $\kappa$ along $\alpha$ is a $(r+n \Delta(\kappa, \alpha) t, s+n \Delta(\kappa, \alpha) v)$ curve, where as in Definition 2.9 . $\Delta$ represents the geometric intersection number in $T$. That is, in the notation of section $2 \tau(\alpha, *, n)=$ $\tau(r+n \Delta(\kappa, \alpha) t, s+n \Delta(\kappa, \alpha) v)$.

We are now in a position to prove the main result

Theorem 6.8. Let $M$ be a handlebody of genus $g>1$. For every positive integer $N$ the following hold.

1. There are infinitely many knots $K \subseteq M$ such that $K$ admits a nontrivial handlebody surgery and

$$
b_{g}(K) \geq N \text {. }
$$

Furthermore, the knots may be taken to have the same genus $g$ bridge number. 
2. There are infinitely many pairs of relatively prime integers $p$ and $q$ such that for each pair, there are infinitely many knots $K \subseteq M$ admitting a surgery yielding a $D(p, q)$-Seifert space in union with $(g-1)$ 1-handles. Furthermore, for each such $K$

$$
b_{g}(K) \geq N
$$

Finally, fixing $(p, q)$, the knots may be taken to have the same genus g bridge number.

The knots in each family above have exteriors in $M$ which are irreducible, boundary-irreducible, atoroidal, and anannular. The slope of each surgery is longitudinal, that is, intersecting the meridian once, and each knot admits exactly one nontrivial $\partial$-reducing surgery.

Remark 6.9. The common bridge number for each family above is not prescribed, it is only known to be bigger than $N$.

Proof. Let $N_{\text {strong }}$ be the constant of Proposition 5.19 for the given $g$. Let $N$ be any given integer. Fix a relatively prime pair of nonnegative integers $r, s$ with $r>s$, and let $\kappa=\tau(r, s)$ and $\alpha=\nu=\tau(1,1)$. Let $C(g, \alpha, \kappa)$ be as in Proposition 6.7 and take $n$ large enough so that this proposition guarantees that

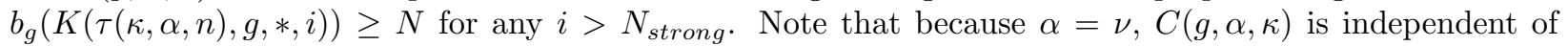

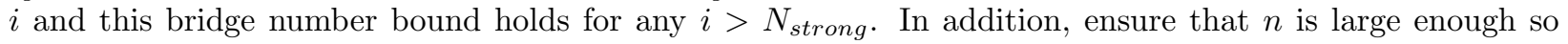
that $\tau(\kappa, \alpha, n)=\tau(r+n \Delta(\kappa, \alpha), s+n \Delta(\kappa, \alpha))=\tau((n+1) r-n s, n r-(n-1) s)$ is not isotopic in $T$ to any of $\{\lambda, \mu, \nu, \lambda-\mu, \lambda+\nu, \mu+\nu\}$ (e.g. by ensuring that $\max (|(n+1) r-n s|,|n r-(n-1) s|)>2$ ). As the disk-hitting number is a knot invariant, Proposition 5.18 (4) shows that, fixing $n$ as above, as $i$ grows there are infinitely many different knots $K(\tau(\kappa, \alpha, n), g, *, i)$ in the genus $g$ handlebody $M \cong M(g, *, i)$. Proposition 5.18(3) shows that all of the knots $K(\tau(\kappa, \alpha, n), g, *, i)$ have a common upper bound on their bridge number, hence we may take an infinite subcollection that has the same bridge number. Finally, as long as we take $|i|>N_{\text {strong }}$, Proposition 5.19 shows that the exteriors of these knots are irreducible, boundary-irreducible, atoroidal, and anannular.

When $*=H$, Proposition 5.18(1) shows that these knots admit longitudinal handlebody surgeries. When $*=S$, Proposition $5.18(2)$ shows that these knots admit a longitudinal surgery which is a $D((n+1) r-$ $n s, n r-(n-1) s)$-Seifert space with $(g-1) 1$-handles attached. (Note that in the case $*=S$, we can arrange different $D((n+1) r-n s, n r-(n-1) s)$-Seifert spaces with $(g-1) 1$-handles attached to be obtained as the result of Dehn surgery on knots in $M$ with bridge number bigger than $N$ as claimed by taking different nonnegative integers $r, s$ or larger integers $n$.)

Finally, we show that each such knot constructed admits exactly one nontrivial boundary-reducing surgery. This argument is adapted from [Bow13, Lemma 5.6 and Proposition 5.7]. Assume the above longitudinal, $\partial$-reducing surgery on $K=K(\tau(\kappa, \alpha, n), g, *, i)$ occurs along the slope $\sigma$. Suppose that there is a second nontrivial boundary-reducible surgery on $K$ in $M$ with slope $\rho \neq \sigma$, so that $M(\rho) \cong H^{\prime} \cup_{P} H(\rho)$. The first paragraph of the proof of Proposition 5.19 shows that, by our choice of $P \subset \partial H^{\prime}$ (with $|i|>N_{\text {strong }}$ ), the curve $\partial_{0} P$ is 3-disk-busting in $H^{\prime}$. Hence any $\partial$-reducing disk for $M(\rho)$ is either contained in $H(\rho)$ (and disjoint from $P$ ) or contains a $\partial$-compressing disk for $P$ among the components of its intersection with $H(\rho)$. Thus we may focus our attention upon $H(\rho)$. Therefore we restrict attention to $\rho$-surgery upon $K \subset H$ and identify $K$ with $\tau=\tau(\kappa, \alpha, n)$.

By Proposition 3.12 and a result of $\mathrm{Wu}$ Wu92b], since $\tau \notin\{\lambda, \mu, \nu\}$, this third surgery slope $\rho$ must have distance one from $\sigma$ and from the meridional slope of $K$. Therefore, recalling Figure 1 since $H \cong T \times I$ and $K$ lies in $T \times\{0\}$ with framing $\sigma$, the effect of $\rho$-surgery on $K$ in $H$ may be viewed as altering the gluing between $T \times[-1,0]$ and $T \times[0,1]$ by a twist along $K$. In particular, we may identify $H(\rho)$ with $H=T \times[-1,1]$ except where $P \subset \partial(T \times[-1,1])$ has been altered by a Dehn twist along $K \times\{1\}$. Thus any essential simple closed curve in $T \times\{ \pm 1\} \subset \partial H$ continues to be a primitive curve in $H(\rho)$. Notably, the curves $\partial P_{ \pm}$are each primitive in $H(\rho)$. 
By a result of Ni Ni11, if $\rho$-surgery on $K$ in $H=P \times I$ preserves the product structure (i.e. $P$ continues to be a fiber in $H(\rho) \cong P \times I)$, then $K$ meets every non-separating $\partial$-compressing disk for $P$ in $H$ at most twice. Indeed this would imply $\tau \in\{\lambda, \mu, \nu, \lambda-\mu, \lambda+\nu, \mu+\nu\}$, cf. Lemmas 2.10 and 2.13 . Above, we have ensured that $\tau(\kappa, \alpha, n)$ does not belong to this set. Hence $H(\rho)$ does not have a product structure with $P$ as a fiber.

If $P$ is $\partial$-compressible in $H(\rho)$, then since $H(\rho)$ is not a product with fiber $P$ and the curves $\partial_{ \pm} P$ are primitive curves, Bow13, Lemma 5.6] implies that $\partial_{0} P$ is not primitive and every $\partial$-compressing disk for $P$ in $H(\rho)$ is disjoint from $\partial_{0} P$. Since $\partial_{0} P$ would be isotopic to a boundary component of an annulus resulting from such a $\partial$-compression of $P$, Bow13, Proposition 5.5.] implies that $\partial_{0} P$ would have to be primitive in either $H^{\prime}$ or $H(\rho)$. However, neither of these can occur since it is 3-disk-busting in $H^{\prime}$ and non-primitive in $H(\rho)$. Thus $P$ is not $\partial$-compressible.

Finally, if the complementary pair of pants $\partial H(\rho)-P$ compresses in $H(\rho)$, then since the curves $\partial_{ \pm} P$ are primitive, it must be that $\partial_{0} P$ bounds a compressing disk. However, in the expression of $H(\rho)$ as $T \times I$ with $P$ Dehn twisted once along $K \times\{1\}, \partial_{0} P$ traverses the annulus $(\partial T) \times I$ twice. Hence the compressing disk with boundary $\partial_{0} P$ is a product disk and thus the curves $\partial_{ \pm} P$ in $T \times\{ \pm 1\}$ project to the same curve in $T$. Yet since $\partial_{ \pm} P$ in $H$ projected to curves $\lambda$ and $\mu$ in $T$, this implies that $K$ must be isotopic to $\nu=\lambda+\mu$ or $\lambda-\mu$.

This finishes the proof that there is exactly one nontrivial boundary-reducible surgery on $K \subset M$.

If we are not concerned about determining a lower bound on bridge number, the following points out that for any $(p, q)$, we can obtain the boundary connected sum of a $D(p, q)$-Seifert space with a genus $g-1-$ handlebody by Dehn surgery on infinitely many knots in a genus $g$ handlebody.

Theorem 6.10. Let $M$ be a handlebody of genus $g>1$ and $p, q$ be non-zero, relatively prime integers. There are infinitely many knots $K \subseteq M$ admitting a longitudinal surgery yielding a $D(p, q)$-Seifert space with $(g-1) 1$-handles attached. Furthermore, the exterior in $M$ of each knot is irreducible, boundaryirreducible, atoroidal, and anannular. Finally, for fixed $p, q$, these knots can be taken to have the same genus $g$ bridge number in $M$.

Proof. If either $|p|=1$ or $|q|=1$ then the $D(p, q)$-Seifert space is a solid torus and this becomes a special case of Theorem 6.8(1). So we assume $|p|,|q|>1$. Then $\tau(p, q) \notin\{\lambda, \mu, \nu, \lambda-\mu, \lambda+\nu, \mu+\nu\}$. Proposition 5.18(2) shows that $K(\tau(p, q), g, S, i)$ admits a surgery which is a $D(p, q)$-Seifert space union $g-1$-handles. As the disk-hitting number is a knot invariant, Proposition 5.18(4) shows that as $i$ grows there are infinitely many such knots. Proposition 5.18(3) shows that all of these have a common upper bound on their bridge number, hence can be taken to have the same bridge number. Finally, as long as we take $|i|>N_{\text {strong }}$, Proposition 5.19 shows that the exteriors of these knots are irreducible, boundary-irreducible, atoroidal, and anannuluar.

\section{The genus two case}

In this section we clarify the connection between the knots in genus 2 handlebodies constructed here and those of [Bow13]. Proposition 6.7 considerably strengthens the results of [Bow13] in certain cases. It identifies new infinite subcollections of knots constructed in Bow13 that have bridge number bigger than one. In fact, it shows these bridge numbers can be taken to be arbitrarily large. This is the content of Corollary 7.3 below. We finish by exploring a technique for producing handlebody/pants pairs that are of strong handlebody or strong Seifert type. By Definition 3.7, and Propositions 3.8, 3.12, these handlebody/pants pairs give rise to lots of knots in a genus 2 handlebody with boundary-reducing surgeries whose exteriors are irreducible, boundary-irreducible, atoroidal, and anannular. Note that subfamilies of these knots can be generated by 
annulus twisting, and the results of [BGL13] can often be applied to establish lower bounds on bridge number - as is done with the knots $K(\tau(\kappa, \alpha, n), 2, *, i)$ in section 6 .

We first show how 2-bridge knot or link exteriors give rise to pairs $\left(H^{\prime}, P^{\prime}\right)$ of handlebody or Seifert type (see Definitions 3.4, 3.5.).

Definition 7.1. Let $J$ be a non-trivial 2-bridge knot or link in canonical 2-bridge position with respect to some height function $h: S^{3} \rightarrow \mathbb{R}$. Then $J$ has an unknotting tunnel connecting its two maxima, i.e. an $\operatorname{arc} t$ such that $H_{J}^{\prime}=S_{J \cup t}^{3}$ is a handlebody. Furthermore, choose $t$ so that it has one maximum and no minimum under $h$ in its interior. The link exterior $S_{J}^{3}$ is obtained from $H_{J}^{\prime}$ by attaching a 2-handle along an attaching curve $c \subseteq \partial H^{\prime}$. Let $P_{J}^{\prime}$ be the pair of pants embedded in $\partial H_{J}^{\prime}$ as shown in Figure 11, so that $\partial P_{J}^{\prime}=a \cup b \cup c$ where $a$ and $b$ are meridians of one component of $J$.

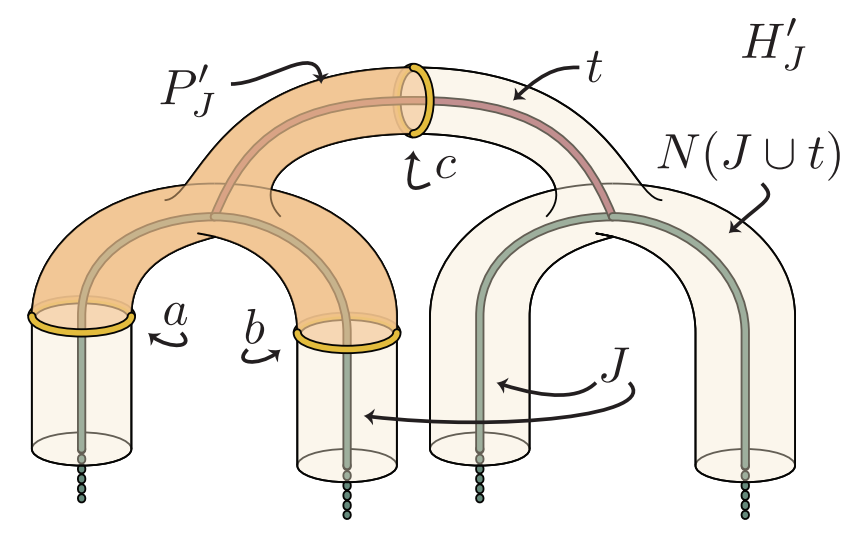

Figure 11: Part of the handlebody $H_{J}^{\prime}=S^{3}-N(J \cup t)$ together with $P_{J}^{\prime}$

Theorem 7.2. Let $H^{\prime}$ be a genus two handlebody and the pair $\left(H^{\prime}, P^{\prime}\right)$ be of handlebody type. Then there is a 2-bridge knot $J$ such that $\left(H^{\prime}, P^{\prime}\right)$ and $\left(H_{J}^{\prime}, P_{J}^{\prime}\right)$ are homeomorphic as pairs.

In particular, let $\left(H_{2}, P(2, H, i)\right)$ be as in section 5.4. Then for each $i$ there is a 2-bridge knot $J_{i}$ such that $\left(H_{2}, P(2, H, i)\right)$ is $\left(H_{J_{i}}^{\prime}, P_{J_{i}}^{\prime}\right)$. The handlebody/knot pair $(M(2, H, i), K(\tau(p, q), 2, H, i))$ is then the pair $\left(M, K_{q, p}^{J_{i}}\right)$ constructed in [Bow13].

Proof. First suppose that $\left(H^{\prime}, P^{\prime}\right)$ is of handlebody type. Let $\partial P^{\prime}=a \cup b \cup c$ where $a, b$ are jointly primitive in $H^{\prime}$. Attach 2-handles to $H^{\prime}$ along $a$ and $b$, and let $a^{\prime}$ and $b^{\prime}$ be the cocores of the handles. Because $a, b$ are jointly primitive, the $\operatorname{arcs} a^{\prime}$ and $b^{\prime}$ in the resulting ball form a rational tangle. Attaching a 2 -handle to $H^{\prime}$ along $c$ gives the exterior of 2-bridge knot, $J$, corresponding to capping the tangle with two unknotted arcs (bridge arcs) outside the tangle. The cocore of the attached 2-handle is an unknotting tunnel for the exterior of $J$ connecting the two bridge arcs. That is, $\left(H^{\prime}, P^{\prime}\right)$ is homeomorphic to $\left(H_{J}^{\prime}, P_{J}^{\prime}\right)$. Having made this identification, the construction of the pair $(M(2, H, i), K(\tau(p, q), 2, H, i))$ is the same as for the pair $\left(M, K_{q, p}^{J_{i}}\right)$ in Bow13. Here we are using the convention that $(\mu, \lambda)$ of $T$ in section 2 corresponds to $(m, l)$ of section 4 in Bow13.

The knots $K_{r, s}^{L}$ in [Bow13] are knots in a genus 2 handlebody that admit non-trivial handlebody surgeries. It is shown there that for certain parameter values, these knots have bridge number bigger than one in the handlebody. Below, we find new parameter values for which the corresponding knots have bridge number bigger than one. Indeed, the bridge numbers get arbitrarily large.

Corollary 7.3. Let $K_{r, s}^{L}$ be the knots in the genus 2 handlebody $M$ constructed in [Bow13]. Let $k$ be a nonzero integer, and fix a positive integer $N$. Let $N_{\text {strong }}$ be the constant for $g=2$ given by Proposition 5.19. For 
$|i|>N_{\text {strong }}$ there are only finitely many knots in the families $\left\{K_{k n+1, n}^{J_{i}}\right\}$ and $\left\{K_{k n-1, n}^{J_{i}}\right\}$ with bridge number less than $N$ in $M$.

Proof. The knot $K_{k n+1, n}^{J_{i}}$ is the knot $K(\tau(n, k n+1), 2, H, i)=K(\tau(\kappa, \alpha, n), 2, H, i)$ where $\kappa=\lambda$ and $\alpha=\tau(1, k)$. Similarly, the knot $K_{k n-1, n}^{J_{i}}$ is the knot $K(\tau(n, k n-1), 2, H, i)=K(\tau(\kappa, \alpha, n-1), 2, H, i)$ where $\kappa=\tau(1, k-1)$ and $\alpha=\tau(1, k)$. The result now follows from Proposition 6.7

Definition 7.1 gives many examples of pairs $\left(H^{\prime}, P^{\prime}\right)$ which are of strong handlebody or strong Seifert type, and, consequently lots of hyperbolic knots in a genus 2 handlebody with boundary-reducing surgeries (see Definition 3.7, and Propositions 3.8, 3.12.

Theorem 7.4. Let $J$ be a nontrivial 2-bridge knot. The pair $\left(H_{J}^{\prime}, P_{J}^{\prime}\right)$ is of handlebody type. If the exterior of $J$ is anannular then $\left(H_{J}^{\prime}, P_{J}^{\prime}\right)$ is of strong handlebody type.

Let $J$ be a 2-bridge link of two components. The pair $\left(H_{J}^{\prime}, P_{J}^{\prime}\right)$ is of Seifert type. If the exterior of $J$ is irreducible and anannular, then $\left(H_{J}^{\prime}, P_{J}^{\prime}\right)$ is of strong Seifert type.

Proof. Let $J$ be a non-trivial 2-bridge knot and $\left(H_{J}^{\prime}, P_{J}^{\prime}\right)$ be as in Definition 7.1. Attaching a 2-handle to $H_{J}^{\prime}$ along $a$ or $b$, we obtain a new knot exterior for which the associated knot has exactly one maximum and one minimum with respect to $h$. This must be the unknot, and therefore $a$ and $b$ are primitive curves in $\partial H_{J}^{\prime}$. The remaining components of $\partial P_{J}^{\prime}$ become meridians of this unknot exterior, so $a$ and $b$ are in fact jointly primitive. Thus $\left(H_{J}^{\prime}, P_{J}^{\prime}\right)$ is of handlebody type.

When $J$ is a 2-bridge link, $a$ and $b$ are parallel and $P^{\prime}$ is obtained by banding annular neighborhoods of $a$ and $b$ in the complement of the annulus cobounded by $a$ and $b$. As each component of a 2-bridge link is unknotted, $a, b$ are each primitive. Thus $\left(H_{J}^{\prime}, P_{J}^{\prime}\right)$ is of Seifert type.

Assume that $J$ is a 2-bridge knot or link whose exterior, $S_{J}^{3}$, is irreducible, anannular, and has incompressible boundary. That exterior is gotten by attaching a 2-handle along $c$ to $H_{J}^{\prime}$. Assume there is a properly embedded essential disk or annulus $A$ in $H_{J}^{\prime}$ that misses $c$. By the assumption on $S_{J}^{3}$, it must be that all components of $\partial A$ become inessential in $\partial S_{J}^{3}$ after adding a 2-handle along $c$. First assume that $A$ is a disk. If $\partial A$ is non-separating in $\partial H_{J}^{\prime}$, then it is isotopic to $c$. But then capping off $\partial A$ with the added 2-handle gives an essential sphere in $S_{J}^{3}$, which is irreducible. So $\partial A$ is separating in $\partial H_{J}^{\prime}$. Each side of $A$ in $H_{J}^{\prime}$ is a solid torus. In particular, this implies that $S_{J}^{3}$ is a solid torus, contradicting the incompressibility of its boundary. Thus it must be that $A$ is an annulus. If both components are parallel in $\partial H_{J}^{\prime}$, then attaching a 2-handle along $c$ must give a lens space summand ( $A$ is essential in $H_{J}^{\prime}$ ). But this is not possible, as such a submanifold lives in the $S_{J}^{3}$. Thus it must be that one component of $\partial A$ is parallel to $c$ and the other bounds a once-punctured torus in $\partial H_{J}^{\prime}$ containing $c$. But then we can cap off these boundary components in $S_{J}^{3}$ to get a non-separating 2-sphere, a contradiction. Thus there is no such essential disk or annulus missing $c$. We conclude that when $J$ is a knot, $\left(H_{J}^{\prime}, P_{J}^{\prime}\right)$ is of strong handlebody type. When $J$ is a two component link, we have that $\left(H_{J}^{\prime}, P_{J}^{\prime}\right)$ is of strong Seifert type by Lemma 7.5 below.

Lemma 7.5. Assume $J$ is a 2-bridge knot or link which is not the unknot, the Hopf link, or a split link. The surface $P_{J}^{\prime}$ is $\partial$-incompressible in $H_{J}^{\prime}$.

Proof. Suppose that $D$ is a $\partial$-compressing disk for $P_{J}^{\prime}$ in $H_{J}^{\prime}$. If $\partial D$ is non-separating in $P_{J}^{\prime}$, then $\partial D \cap c$ is empty since $c$ is not primitive ( $J$ is not the unknot). So $D$ becomes an essential disk in the link exterior $S_{J}^{3}$, which is impossible by hypothesis.

If $\partial D$ separates $H_{J}^{\prime}$ (so that it also separates $P_{J}^{\prime}$ ), we obtain two solid tori after performing the $\partial$-compression, and each contains an annulus coming from $P_{J}^{\prime}$ in its boundary. If $\partial D$ meets $a$, say, then examine the solid torus with the annulus containing $b$ on its boundary. Since a primitive curve in a handlebody of genus $g>1$ remains primitive after cutting along a disk disjoint from the curve, the annulus containing $b$ must 
be longitudinal in the boundary of its solid torus. However, this implies that there is a non-separating $\partial$-compressing disk for $P_{J}^{\prime}$ and we apply the argument above. A similar argument works if $\partial D$ meets $b$ or $c$.

Suppose then that $\partial D$ separates $P_{J}^{\prime}$ but not $\partial H_{J}^{\prime}$. If $\partial D$ meets $a$ or $b$, we obtain a disk in a link exterior after attaching a 2 -handle along $c$. This disk must be trivial in the exterior of $J$ by hypothesis. Furthermore, since $\partial D$ does not separate $\partial H_{J}^{\prime}$, it must be parallel to $c$. This gives a non-separating sphere in the exterior $S_{J}^{3}$, which is impossible. On the other hand, if $\partial D$ meets $c$, then $D$ extends across $N(t)$ ( $t$ the tunnel of Definition 7.1) to an annulus $A$ properly embedded in $S_{J}^{3}$ one of whose boundary components is a meridian of a component of $J$. Therefore $A$ is incompressible in $S_{J}^{3}$. We may take $t$ to be the cocore of $A$. Since $t$ is not boundary-parallel in $S_{J}^{3}, A$ is also not boundary-parallel. When $J$ is a knot, both boundary components of $A$ are meridians, implying that $J$ is not prime. This is impossible since $J$ is 2 -bridge. On the other hand, when $J$ is a two component link, $A$ shows that $J$ must be the Hopf link, contradicting our hypotheses.

\section{References}

[Ber] John Berge, Knots in handlebodies which can be surgered to produce handlebodies, Unpublished manuscript.

[Ber91] _ The knots in $D^{2} \times S^{1}$ which have nontrivial Dehn surgeries that yield $D^{2} \times S^{1}$, Topology and its Applications 38 (1991), no. 1, 1 - 19.

[BGL13] Kenneth L. Baker, Cameron Gordon, and John Luecke, Bridge number and integral Dehn surgery, 2013, Alg. Geom. Top., To appear.

[Bow13] R. Sean Bowman, Knots in handlebodies with nontrivial handlebody surgeries, J. Topology 6 (2013), no. 4, 955-981.

[CGLS87] M. Culler, C. McA. Gordon, J. Luecke, and P. B. Shalen, Dehn surgery on knots, Ann. Math. 125 (1987), 237-300.

[FMP03] Roberto Frigerio, Bruno Martelli, and Carlo Petronio, Dehn filling of cusped hyperbolic 3manifolds with geodesic boundary, J. Differential Geom. 64 (2003), no. 3, 425-455. MR 2032111 (2005d:57024)

[Gab89] David Gabai, Surgery on knots in solid tori, Topology 28 (1989), no. 1, 1-6. MR 991095 (90h:57005)

[Gab90]_, 1-Bridge braids in solid tori, Topology Appl. 37 (1990), no. 3, 221-235.

[GL89] C. McA. Gordon and J. Luecke, Knots are determined by their complements, J. Amer. Math. Soc. 2 (1989), no. 2, 371-415. MR 965210 (90a:57006a)

[Gor87] C. McA. Gordon, On primitive sets of loops in the boundary of a handlebody, Topology Appl. 27 (1987), no. 3, 285-299. MR 918538 (88k:57013)

[Gor98] _ Boundary slopes of punctured tori in 3-manifolds, Trans. Amer. Math. Soc. 350 (1998), no. $5,1713-1790$.

[Ni11] Yi Ni, Dehn surgeries on knots in product manifolds, Journal of Topology 4 (2011), no. 4, 799-816.

[Sch90] Martin Scharlemann, Producing reducible 3-manifolds by surgery on a knot, Topology 29 (1990), no. 4, 481-500. MR 1071370 (91i:57003)

[Wu92a] Ying Qing Wu, A generalization of the handle addition theorem, Proc. Amer. Math. Soc. 114 (1992), no. 1, 237-242. MR 1070535 (92c:57018) 
[Wu92b] _ Incompressibility of surfaces in surgered 3-manifolds, Topology 31 (1992), no. 2, 271-279. MR 1167169 (94e:57027)

[Wu93]_ D-reducing Dehn surgeries and 1-bridge knots, Math. Ann. 295 (1993), no. 2, 319-331. MR 1202395 (94a:57036)

[Yos14] Michael Yoshizawa, High distance Heegaard splittings via Dehn twists, Algebr. Geom. Topol. 14 (2014), no. 2, 979-1004. MR 3180825 